\title{
K-theory, cyclic cohomology and pairings for quantum Heisenberg manifolds
}

\author{
Olivier Gabriel
}

\begin{abstract}
The $\mathrm{C}^{*}$-algebras called quantum Heisenberg manifolds (QHMs) were introduced by Rieffel in 1989 as strict deformation quantizations of Heisenberg manifolds. It was later shown that they are also examples of generalized crossed products. In this article, we compute the pairings of K-theory and cyclic cohomology on the QHM. Combining these calculations with other results proved elsewhere, we also determine the periodic cyclic homology and cohomology of these algebras, and obtain explicit bases of the periodic cyclic cohomology of the QHM. We further isolate bases of periodic cyclic homology, expressed as Chern characters of the K-theory.
\end{abstract}

Mathematics Subject Classification (2010). 46L87, 19K99.

Keywords. Quantum Heisenberg manifolds, K-theory, cyclic cohomology, Chern-Connes pairings, generalized crossed product, Pimsner algebra, Heisenberg group.

\section{Introduction}

Quantum Heisenberg manifolds (QHMs) were introduced by Rieffel in [31]. They constitute a family of $\mathrm{C}^{*}$-algebras $D_{\mu, v}^{c}$ indexed by $c \in \mathbb{Z}$ and $\mu, v \in \mathbb{R}$. In the following, we denote these algebras by $D$ for brevity. In the article [5] by Abadie, Eilers and Exel, it was proved that QHMs are generalized crossed products. One can show that QHMs are also Pimsner algebras, a closely related notion which was introduced in the seminal article by Pimsner [28]. Since this latter class has been more widely studied (see for example [29], [17], [22], [19]), let us mention that both notions often coincide and that a common generalization can be found (see [24]).

QHMs have been closely studied in series of articles by Abadie (see for instance [2], [3], [6], [4]) and Chakraborty (see [10] and [9]). In [14], Connes and DuboisViolette related QHMs to the noncommutative 3-spheres they introduced in [13]. More recently, Kang studied these algebras through their Yang-Mills functionals ([23]) and Kumjian started considering them as groupoids algebras ([1]).

The present article is a refinement of the previous studies of QHMs by means of pairings between cyclic cohomology and K-theory. These pairings were first defined 
by Connes in his article [11] of 1985. They have a parity: odd cocycles pair with $K_{1}$ and even cocycles with $K_{0}$.

After reviewing QHMs and the associated Heisenberg group action, we define a smooth subalgebra $\mathcal{D}$ of $D$. Using the general Proposition 3.5, we then construct explicit cyclic cocycles $\left(\varphi_{i}\right)_{i=1,2,3},\left(\varphi_{i, 3}\right)_{i=1,2}$ and $\varphi_{1,2,3}$ out of the Lie group action (see Proposition 3.8). We rely on previous work by Abadie to obtain finitely generated projective modules $\mathcal{N}, \mathcal{N}^{\dagger}$ over $\mathcal{D}$. We then construct a generating set $\left(U_{1}, U_{2}, U_{3}\right)$ of $K_{1}(\mathcal{D}) \otimes_{\mathbb{Z}} \mathbb{C}$.

There are two main results. The first one consists of the Theorems 8.3 and 8.4:

Theorem. Taking the notations of Proposition 3.8 and Section 5,

- the family $\left(\tau, \varphi_{1,3}, \varphi_{2,3}\right)$ is a basis of $\mathrm{HP}^{0}(\mathcal{D})$;

- the family $\left(\varphi_{1}, \varphi_{2}, \varphi_{1,2,3}\right)$ is a basis of $\operatorname{HP}^{1}(\mathcal{D})$;

- the family $\left(\mathrm{Ch}\left(U_{1}\right), \mathrm{Ch}\left(U_{2}\right), \mathrm{Ch}\left(U_{3}\right)\right)$ is a basis of $\mathrm{HP}_{1}(\mathcal{D})$.

Moreover, if $\mu \neq 0 \neq v$, then the family $\left(\operatorname{Ch}\left(\mathcal{D}_{\mu, v}^{c}\right), \operatorname{Ch}\left(\mathcal{N}_{\mu, \nu}^{c}\right), \operatorname{Ch}\left(\mathcal{N}_{\mu, \nu}^{\dagger c}\right)\right)$ is a basis of $\mathrm{HP}_{0}\left(\mathcal{D}_{\mu, v}^{c}\right)$.

Notice that this theorem relies on $k k$-equivalences that are constructed in [20]. The proof also depends on Tables 1 and 2 that give the pairings of cyclic cohomology with $\mathrm{K}$-theory in the even and odd cases:

Table 1

\begin{tabular}{|l|c|c|c|}
\cline { 2 - 4 } \multicolumn{1}{c|}{} & $\tau$ & $\varphi_{1,3}$ & $\varphi_{2,3}$ \\
\hline$[\mathcal{D}]$ & 1 & 0 & 0 \\
\hline$[\mathcal{N}]$ & $2 \mu$ & $-i 2 \pi$ & 0 \\
\hline$\left[\mathcal{N}^{\dagger}\right]$ & $2 v$ & 0 & $i 2 \pi$ \\
\hline
\end{tabular}

Table 2

\begin{tabular}{|c|c|c|c|}
\cline { 2 - 4 } \multicolumn{1}{c|}{} & $\varphi_{1}$ & $\varphi_{2}$ & $\varphi_{1,2,3}$ \\
\hline$\left[U_{1}\right]$ & $-\sqrt{i 2 \pi}$ & 0 & 0 \\
\hline$\left[U_{2}\right]$ & 0 & $-\sqrt{i 2 \pi}$ & 0 \\
\hline$\left[U_{3}\right]$ & $-\sqrt{i 2 \pi} 2 c \nu$ & $\sqrt{i 2 \pi} 2 c \mu$ & $-(i 2 \pi)^{3 / 2} c / 3$ \\
\hline
\end{tabular}

The computation of these tables is the second main result.

This paper is organized as follows. In Section 2, we review the properties of QHMs. Section 3 is devoted to the construction of cyclic cocycles. The pairings in the even cases are computed in Section 4. Sections 5 and 6 are concerned with the calculation in the odd cases. Section 7 focuses on unfolding the consequences of the previous computations. The computation of the periodic homology and cohomology is completed in Section 8. We conclude in Section 9 with various remarks and perspectives. 


\section{Quick review of QHMs}

We will define QHMs as generalized crossed products of $A=C\left(T^{2}\right)$ by certain Hilbert bimodules $M_{\mu, v}^{c}$. General references for Hilbert modules are [25], [7].

Several definitions of "Hilbert bimodules" exist in the literature, we use the original Definition 1.8 of [8]:

Definition 2.1 (Hilbert bimodule). A $A$-A-Hilbert bimodule $E$ is a vector space such that

- $E$ is a right Hilbert $A$-module with $A$-valued scalar product $\langle,\rangle_{A}$,

- $E$ is a left Hilbert $A$-module with $A$-valued scalar product ${ }_{A}\langle$,$\rangle ,$

- the scalar products are compatible in the sense that $\xi\langle\zeta, \eta\rangle_{A}={ }_{A}\langle\xi, \zeta\rangle \eta$.

Note that the above definition is not the definition used by Pimsner in his paper [28].

Definition 2.2 is closely related to Theorem 3.4 of [28]. However, since we consider a more special case here, we refer to [8], Definition 2.1, and [18], Definition 4.5:

Definition 2.2 (Covariant representation). Let $E$ be an $A$ - $A$ Hilbert bimodule. A covariant representation of $E$ on a $C^{*}$-algebra $B$ is a pair $(\pi, \mathcal{T})$ which consists of

- a $*$-homomorphism of algebras $\pi: A \rightarrow B$,

- a linear map $\mathcal{T}: E \rightarrow B$ satisfying

$$
\begin{aligned}
& \text { (i) } \mathcal{T}(\xi)^{*} \mathcal{T}(\zeta)=\pi\left(\langle\xi, \zeta\rangle_{A}\right), \quad \text { (ii) } \quad \mathcal{T}(\xi) \pi(a)=\mathcal{T}(\xi a) \text {, } \\
& \text { (iii) } \quad \pi(a) \mathcal{T}(\xi)=\mathcal{T}(a \xi), \quad \text { (iv) } \mathcal{T}(\xi) \mathcal{T}(\zeta)^{*}=\pi\left({ }_{A}\langle\xi, \zeta\rangle\right) \text {. }
\end{aligned}
$$

We want to construct a universal $\mathrm{C}^{*}$-algebra out of these representations. First notice that for any covariant representation $(\pi, \mathcal{T})$,

$$
\|\mathcal{T}(\xi)\|^{2}=\left\|\mathcal{T}(\xi)^{*} \mathcal{T}(\xi)\right\|=\left\|\pi\left(\langle\xi, \xi\rangle_{A}\right)\right\| \leqslant\|\xi\|^{2} \quad \text { for all } \xi \in E,
$$

using the Hilbert module norm $\|\xi\|$. Now, given an arbitrary Hilbert bimodule $E$ over a $C^{*}$-algebra $A$, Proposition 2.3 of [5] shows that such covariant representations exist. Together, these properties justify the following definition.

Definition 2.3 (Generalized crossed product). Let $E$ be an $A$ - $A$ Hilbert bimodule. The generalized crossed product $A \rtimes_{E} \mathbb{Z}$ of $A$ by $E$ is the universal $C^{*}$-algebra generated by the covariant representations of $E$.

We now define the Hilbert bimodule $M_{\mu, \nu}^{c}$. 
Definition 2.4 (Hilbert bimodule over $A$ ). Given two real numbers $\mu, v$ and an integer $c>0$, we define a Hilbert bimodule $M_{\mu, \nu}^{c}$ over $A=C\left(T^{2}\right)$ as the set of continuous functions $\xi: \mathbb{R} \times S^{1} \rightarrow \mathbb{C}$ which satisfy

$$
\xi(x+1, y)=e(-c(y-v)), \quad \xi(x, y) \xi(x, y+1)=\xi(x, y),
$$

where $e(x)=e^{2 \pi i x}$. We give it a bimodule structure by (direct) pointwise multiplication on the left and right multiplication defined by $(\xi \cdot a)(x, y)=\xi(x, y) a(x-$ $2 \mu, y-2 v)$. We denote by $\sigma$ the automorphism of $A$ defined by $\sigma(a)(x, y)=$ $a(x-2 \mu, y-2 v)$. The definitions of left and right actions entail

$$
\xi a=\sigma(a) \xi .
$$

for all $\xi \in M_{\mu, v}^{c}$ and all $a \in A$. Finally, the scalar products are

$$
\begin{aligned}
& \left\langle\xi_{1}, \xi_{2}\right\rangle_{A}(x, y)=\overline{\xi_{1}(x+2 \mu, y+2 v)} \xi_{2}(x+2 \mu, y+2 v), \\
& { }_{A}\left\langle\xi_{1}, \xi_{2}\right\rangle(x, y)=\xi_{1}(x, y) \overline{\xi_{2}(x, y)} .
\end{aligned}
$$

We can easily verify that this indeed defines a Hilbert bimodule. For convenience, we will use the shorthand notation $M$ instead of $M_{\mu, v}^{c}$. We are now ready to define the QHMs.

Definition 2.5 (Quantum Heisenberg manifolds). Given an integer $c>0$ and $\mu, \nu \in$ $\mathbb{R}$, the quantum Heisenberg manifold $D_{\mu, v}^{c}$ is the generalized crossed product $A \rtimes_{M_{\mu, \nu}^{c}} \mathbb{Z}$ of $A$ by $M_{\mu, \nu}^{c}$.

In the following, we will write $D$ instead of $D_{\mu, v}^{c}$ whenever possible. We will identify the elements $\xi \in M$ with their images in $D$. From the definition of $D$ it follows that

$$
{ }_{A}\langle\xi, \zeta\rangle=\xi \zeta^{*}, \quad\langle\xi, \zeta\rangle_{A}=\xi^{*} \zeta
$$

for any $\xi, \zeta \in M$. A more explicit definition was given in [31], Theorem 5.5. However, we will use another definition (see [5], Example 3.3): $D$ is the completion of the algebra

$$
D_{0}=\left\{F \in C_{\mathrm{c}}\left(\mathbb{Z} \rightarrow C_{\mathrm{b}}\left(\mathbb{R} \times S^{1}\right)\right) \mid F(p, x+1, y)=e(-c p(y-p v)) F(p, x, y)\right\}
$$

equipped with multiplication

$$
\left(F_{1} \cdot F_{2}\right)(p, x, y)=\sum_{q \in \mathbb{Z}} F_{1}(q, x, y) F_{2}(p-q, x-q 2 \mu, y-q 2 v)
$$

and involution

$$
F^{*}(p, x, y)=\bar{F}(-p, x-2 p \mu, y-2 p v) .
$$

Like every generalized crossed product, $D$ is endowed with a gradation by $\mathbb{Z}: a \in$ $A \subseteq D$ has degree 0 and $\xi \in M \subseteq D$ has degree 1 . 
Proposition 2.6. For all $M_{\mu, v}^{c}$, we can find $\xi_{1}, \xi_{2} \in M_{\mu, v}^{c}$ such that

$$
\sum_{i=1}^{2} \xi_{i}^{*} \xi_{i}=\sum_{i=1}^{2}\left\langle\xi_{i}, \xi_{i}\right\rangle_{A}=1, \quad \sum_{i=1}^{2} \xi_{i} \xi_{i}^{*}=\sum_{i=1}^{2}{ }_{A}\left\langle\xi_{i}, \xi_{i}\right\rangle=1
$$

Proof. Let $U_{1}, U_{2}$ be the respective images of $\left.V_{1}=\right]-1 / 3,1 / 3\left[\right.$ and $\left.V_{2}=\right] 1 / 6,5 / 6[$ by the quotient map $\pi: \mathbb{R} \rightarrow \mathbb{R} / \mathbb{Z} \simeq S^{1}$. Then $U_{1}, U_{2}$ is an open cover of $S^{1}$. We call

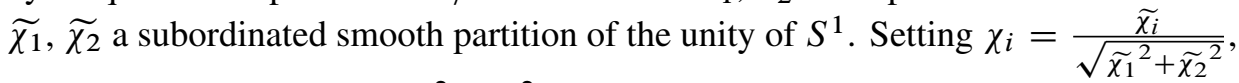
we get $\chi_{1}$ and $\chi_{2}$ such that $\chi_{1}^{2}+\chi_{2}^{2}=1$ and Supp $\chi_{i} \subseteq U_{i}$.

To define $\xi_{1}$ on the cylinder $\mathbb{R} \times S^{1}$, we first set $\xi_{1}(x, y)=\chi_{1}(x)$ on $[-1 / 2,1 / 2] \times$ $S^{1}$. This function can then be extended to the whole $\mathbb{R} \times S^{1}$ by enforcing the equations (2.1). Notice that the extension is possible because $\xi_{1}$ vanishes on the boundaries of $[-1 / 2,1 / 2] \times S^{1}$.

The same process can be applied to $[0,1] \times S^{1}$ to define $\xi_{2}$. In the end, we get $\xi_{i}$ such that

$$
\left\langle\xi_{i}, \xi_{i}\right\rangle_{A}(x, y)=\chi_{i}(x+2 \mu, y+2 v) \overline{\chi_{i}(x+2 \mu, y+2 v)}=\chi_{i}^{2}(x+2 \mu, y+2 v) .
$$

The first equation of (2.3) hence comes from the property of the $\chi_{i}$. The same kind of computation provides the second equation.

Definition 2.7 (Heisenberg group $H_{1}$ ). The Heisenberg group $H_{1}$ is the subgroup of $\mathrm{GL}_{3}(\mathbb{R})$ of the matrices

$$
\left(\begin{array}{lll}
1 & s & t \\
0 & 1 & r \\
0 & 0 & 1
\end{array}\right), \quad r, s, t \in \mathbb{R}
$$

Rieffel actually uses another parametrization of $H_{1}$, see [31], p. 539. He takes a nonzero $c$ and identifies $H_{1}$ with $\mathbb{R}^{3}$ equipped with the product

$$
\left(r^{\prime}, s^{\prime}, t^{\prime}\right)(r, s, t)=\left(r^{\prime}+r, s^{\prime}+s, t^{\prime}+t+c s^{\prime} r\right) .
$$

The following definition has its origin in [31], Proposition 5.6.

Definition 2.8 (Action of the Heisenberg group). There is a pointwise continuous action of $H_{1}$ on $D$ defined on $D_{0}$ by

$$
\alpha_{(r, s, t)}(F)(p, x, y)=e(-p(t+c s(x-r))) F(p, x-r, y-s) .
$$

Using parameters $(r, s, t)$, the infinitesimal generators of this action are

$$
\begin{aligned}
\partial_{1} F(p, x, y) & =-\frac{\partial F}{\partial x}(p, x, y), \\
\partial_{3}(F)(p, x, y) & =-i 2 \pi p F(p, x, y), \\
\partial_{2}(F)(p, x, y) & =-\frac{\partial F}{\partial y}(p, x, y)-i 2 \pi p c x F(p, x, y) .
\end{aligned}
$$


They fulfill the commutation relations

$$
\left[\partial_{1}, \partial_{2}\right]=-c \partial_{3}, \quad\left[\partial_{1}, \partial_{3}\right]=0, \quad\left[\partial_{2}, \partial_{3}\right]=0
$$

Definition 2.9 (Trace on QHM). A trace $\tau$ is given by $\tau(F)=\int_{0}^{1} \int_{S^{1}} F(0, x, y) d y d x$. It is invariant under the action of $H_{1}$.

Finally, the K-theory of the QHM was computed in [3] (Theorem 3.4):

Theorem 2.10 (Abadie, 1995). $K_{0}(D)=\mathbb{Z}^{3} \oplus \mathbb{Z} / c \mathbb{Z}$ and $K_{1}(D)=\mathbb{Z}^{3}$.

\section{Cyclic cocycles and Lie groups actions}

We start this section by gathering definitions and results about cyclic cohomology. We follow the exposition of [12]. The definition of cyclic cohomology is given in [12], III.1, p. 182:

Definition 3.1 (Cyclic cohomology). Given an algebra $\mathcal{A}$, the cyclic cohomology $\operatorname{HC}^{n}(\mathcal{A})$ is the cohomology of the complex $\left(C_{\lambda}^{n}, b\right)$ where $C_{\lambda}^{n}$ is the space of $(n+1)$ linear forms $\phi$ on $\mathcal{A}$ such that

$$
\phi\left(a^{0}, a^{1}, \ldots, a^{n}\right)=(-1)^{n} \phi\left(a^{n}, a^{0}, \ldots, a^{n}\right)
$$

and the coboundary map $b$ is given by

$$
\begin{aligned}
b \phi\left(a^{0}, \ldots, a^{n}, a^{n+1}\right)= & \sum_{j=0}^{n}(-1)^{j} \phi\left(a^{0}, \ldots, a^{j} a^{j+1}, \ldots, a^{n+1}\right) \\
& +(-1)^{n+1} \phi\left(a^{n+1} a^{0}, \ldots, a^{n}\right) .
\end{aligned}
$$

The set of all closed cochains in the above sense is denoted by $Z C^{n}(\mathcal{A})$. Its elements are called cyclic cocycles.

Definition 3.2 (Cycle). A cycle of dimension $n$ is a triple $\left(\Omega, d, \int\right)$ where $\Omega=$ $\bigoplus_{j=0}^{n} \Omega^{j}$ is a graded algebra over $\mathbb{C}, d$ is a graded derivation of degree 1 such that $d^{2}=0$ and $\int: \Omega^{n} \rightarrow \mathbb{C}$ is a closed graded trace on $\Omega$.

A cycle over an algebra $\mathcal{A}$ is a cycle $\left(\Omega, d, \int\right)$ together with an homomorphism $\rho: \mathcal{A} \rightarrow \Omega^{0}$. The character of such a cycle is $\phi$, the $(n+1)$-linear form on $\mathcal{A}$ given by

$$
\phi\left(a^{0}, \ldots, a^{n}\right)=\int \rho\left(a^{0}\right) d\left(\rho\left(a^{1}\right)\right) \ldots d\left(\rho\left(a^{n}\right)\right)
$$

The character of a cycle essential defines the cycle. Moreover, we can restate [12], III.1, Proposition 4, p. 186: 
Proposition 3.3. If $\phi$ is an $(n+1)$-linear functional on $\mathcal{A}$, the following conditions are equivalent:

- $\phi$ is the character of a certain $n$-dimensional cycle $\left(\Omega, d, \int\right)$.

- $\phi$ is a cyclic cocycle.

In the following definition and proposition, we adapt the construction of [12], III.6, Example 12 c), p. 254: let $A$ be a Banach algebra equipped with a pointwise continuous action $\alpha$ of the Lie group $G$. We let $\mathcal{A}=\left\{x \in A \mid g \mapsto \alpha_{g}(x) \in\right.$ $\left.C^{\infty}(G \rightarrow A)\right\}$ and $g$ be the Lie algebra associated to $G$.

Definition 3.4 (Differential algebra associated to a lie group action). The differential algebra $\Omega_{G}$ associated with the action $\alpha$ is the graded differential algebra defined by the alternating $\mathcal{A}$-valued multilinear forms on $\mathrm{g}$ equipped with the differential $d$ :

$$
\begin{aligned}
d \omega\left(X_{1}, \ldots, X_{n+1}\right)= & \sum_{i=1}^{n+1}(-1)^{i} X_{i} \omega\left(X_{1}, \ldots, \check{X}_{i}, \ldots, X_{n+1}\right) \\
& +\sum_{i<j}(-1)^{i+j} \omega\left(\left[X_{i}, X_{j}\right], X_{1}, \ldots, \check{X}_{i}, \ldots, \check{X}_{j}, \ldots X_{n+1}\right) .
\end{aligned}
$$

The following proposition is a restatement of the property described in [12], p. 255. We adapt it to our simple case and state it.

Proposition 3.5. If $\tau$ is a $G$-invariant trace over $A$ and $\xi_{1} \wedge \cdots \wedge \xi_{k} \in \Lambda^{k} \mathrm{~g}$ satisfies

$$
\sum_{i<j}(-1)^{i+j}\left[\xi_{i}, \xi_{j}\right] \wedge \xi_{1} \wedge \cdots \wedge \hat{\xi}_{i} \wedge \cdots \wedge \hat{\xi}_{j} \wedge \cdots \wedge \xi_{n}=0,
$$

then

$$
\left(a_{0} \otimes a_{1} \otimes \cdots \otimes a_{n}\right) \mapsto \sum_{\sigma \in \Sigma_{n}} \epsilon(\sigma) \tau\left(a_{0} \xi_{\sigma(1)}\left(a_{1}\right) \ldots \xi_{\sigma(n)}\left(a_{n}\right)\right)
$$

is a cyclic cocycle on $\mathcal{A}$.

In order to use Definition 3.4 and Proposition 3.5, we need

Definition 3.6 (Smooth QHM). The smooth $Q H M \mathcal{D}$ is defined by $\mathcal{D}=\{F \in D \mid$ $\left.g \mapsto \alpha_{g}(F) \in C^{\infty}\left(H_{1} \rightarrow D\right)\right\}$.

Since $\alpha$ is pointwise continuous, Proposition 3.45 of [21], p. 138, shows that $\mathcal{A}$ is dense in $A$ and stable under holomorphic calculus as well. Such algebras are called pre- $C^{*}$-algebras in [21], see Definition 3.26, p. 134. It is a well-known fact that for such subalgebras the injection $\mathcal{A} \hookrightarrow A$ induces an isomorphism in K-theory (see for instance [21], Theorem 3.44, p. 138). Hence we have 
Lemma 3.7. $\mathcal{D}$ is a dense subalgebra that is stable under holomorphic functional calculus and therefore

$$
K_{0}(\mathcal{D})=K_{0}(D)=\mathbb{Z}^{3} \oplus \mathbb{Z} / c \mathbb{Z}, \quad K_{1}(\mathcal{D})=K_{1}(D)=\mathbb{Z}^{3} .
$$

Proposition 3.8. The seven following multilinear forms are cyclic cocycles on $\mathcal{D}$ :

- Degree 0: trace $\tau$.

- Degree 1: $\varphi_{i}$ for $i=1,2,3$ where $\varphi_{i}\left(a_{0}, a_{1}\right)=\tau\left(a_{0} \partial_{i}\left(a_{1}\right)\right)$.

- Degree 2: $\varphi_{1,3}$ and $\varphi_{2,3}$ where

$$
\varphi_{i, 3}\left(a_{0}, a_{1}, a_{2}\right)=\tau\left(a_{0}\left(\partial_{i}\left(a_{1}\right) \partial_{3}\left(a_{2}\right)-\partial_{3}\left(a_{1}\right) \partial_{i}\left(a_{2}\right)\right)\right) .
$$

- Degree 3: $\varphi_{1,2,3}$ given by

$$
\varphi_{1,2,3}\left(a_{0}, a_{1}, a_{2}, a_{3}\right)=\sum_{\sigma \in \Sigma_{3}} \varepsilon(\sigma) \tau\left(a_{0} \partial_{\sigma(1)} a_{1} \partial_{\sigma(2)} a_{2} \partial_{\sigma(3)} a_{3}\right) .
$$

Proof. This is a straightforward application of Proposition 3.5.

Degree 0: There is nothing to check since $\tau$ is a trace.

Degree 1: Any derivation generates a cyclic cocycle.

Degree 2: The commutation relations (2.5) show that $\xi_{1} \wedge \xi_{3}$ and $\xi_{2} \wedge \xi_{3}$ satisfy condition (3.1).

Degree 3: Start with $\xi_{1} \wedge \xi_{2} \wedge \xi_{3}$. The condition can therefore be written

$$
(-1)^{3}\left[\xi_{1}, \xi_{2}\right] \wedge \xi_{3}+(-1)^{4}\left[\xi_{1}, \xi_{3}\right] \wedge \xi_{2}+(-1)^{5}\left[\xi_{2}, \xi_{3}\right] \wedge \xi_{1}=0 .
$$

The commutation relations (2.5) ensure that this expression vanishes.

Remark 3.9. One could expect a third 2-cyclic cocycle $\varphi_{1,2}$ given by

$$
\varphi_{1,2}\left(a_{0}, a_{1}, a_{2}\right)=\tau\left(a_{0}\left(\partial_{1}\left(a_{1}\right) \partial_{2}\left(a_{2}\right)-\partial_{2}\left(a_{1}\right) \partial_{1}\left(a_{2}\right)\right)\right) .
$$

The cocycle $\varphi_{1,2}$ is indeed a Hochschild cocycle, as an easy computation shows. However, Proposition 9.1 implies that $\varphi_{1,2}$ is not a cyclic cocycle.

Proof. Indeed, if $\varphi_{1,2}$ were a cyclic cocycle, then $\varphi_{1,2}\left(1, a_{1}, a_{2}\right)=0$. Yet,

$$
\begin{aligned}
\varphi_{1,2}\left(1, a_{1}, a_{2}\right) & =\tau\left(\partial_{1}\left(a_{1}\right) \partial_{2}\left(a_{2}\right)-\partial_{2}\left(a_{1}\right) \partial_{1}\left(a_{2}\right)\right) \\
& =\tau\left(\partial_{1}\left(a_{1} \partial_{2}\left(a_{2}\right)\right)-\partial_{2}\left(a_{1} \partial_{2}\left(a_{2}\right)\right)\right)-\tau\left(a_{1} \partial_{1} \partial_{2}\left(a_{2}\right)-a_{1} \partial_{2} \partial_{1}\left(a_{2}\right)\right) \\
& =c \tau\left(a_{1} \partial_{3}\left(a_{2}\right)\right) \\
& =c \varphi_{3}\left(a_{1}, a_{2}\right),
\end{aligned}
$$

using (2.5). But Proposition 9.1 shows that $\varphi_{3}$ pairs nontrivially with a Hochschild cocycle and therefore is nonzero. 


\section{Modules and even pairings}

Bear in mind (see [11], part II, Theorem 9) that if $a_{i} \otimes b_{i} \in A \otimes M_{n}(\mathbb{C})$ and $\phi \in \mathrm{HC}^{*}$, the cup product $\phi \# \operatorname{Tr}$ is defined by

$$
(\phi \# \operatorname{Tr})\left(a^{0} \otimes b^{0}, \ldots, a^{n} \otimes b^{n}\right)=\phi\left(a^{0}, \ldots, a^{n}\right) \operatorname{Tr}\left(b^{0} \ldots b^{n}\right) .
$$

We follow the definition and normalizations of [12] (III.3, Proposition 2, p. 224):

Definition 4.1 (Even Chern-Connes pairings). The formula

$$
\langle[e],[\phi]\rangle=\frac{1}{m !}(\phi \# \operatorname{Tr})(e, \ldots, e),
$$

defines a bilinear pairing between $K_{0}(\mathcal{A})$ and $\operatorname{HC}^{2 m}(\mathcal{A})$, where $[e] \in K_{0}(\mathcal{A})$ and $\phi \in Z C^{2 m}(\mathcal{A})$.

Furthermore, there is a periodicity map, $S: \mathrm{HC}^{n}(\mathcal{A}) \rightarrow \mathrm{HC}^{n+2}(\mathcal{A})$, which enables us to define the groups $\operatorname{HP}^{*}(\mathcal{A})$; see [21], 10.1, Definition 10.5, p. 445:

Definition 4.2 (Periodic cyclic cohomology). The periodic cyclic cohomology is composed of two groups obtained as inductive limits:

$$
\operatorname{HP}^{0}(\mathcal{A})=\lim _{\longrightarrow} \operatorname{HC}^{2 k}(\mathcal{A}), \quad \operatorname{HP}^{1}(\mathcal{A})=\lim _{\longrightarrow} \operatorname{HC}^{2 k+1}(\mathcal{A}) .
$$

The above pairings are in fact defined on $\operatorname{HP}^{0}(\mathcal{A})$ because they satisfy $\langle[e],[S \phi]\rangle=$ $\langle[e],[\phi]\rangle$.

Before stating our theorem on even pairings of QHM, we need to introduce elements of $K_{0}(\mathcal{D})$, i.e., finitely generated projective modules over $\mathcal{D}$. For brevity, we will call these finite projective modules.

These modules over $D_{\mu, v}^{c}$ were studied by Abadie in [2] and [3]. We present the results of [2], pp. 2-3:

Theorem 4.3 (Abadie, 1992). For all $c \in \mathbb{N}^{*}, \mu, v \in \mathbb{R}$ such that $\mu^{2}+v^{2} \neq 0$, there is a finite projective right module $N_{\mu, \nu}^{c}$ over $D_{\mu, \nu}^{c} . N_{\mu, \nu}^{c}$ is obtained by completing $C_{\mathrm{c}}\left(\mathbb{R} \times S^{1}\right)$ with respect to the $D_{\mu, v^{-}}^{c}$-valued scalar product:

$$
\langle f, g\rangle_{D_{\mu, v}^{c}}(p, x, y)=\sum_{n \in \mathbb{Z}} e(c n p(y-p v)) \overline{f(x+n, y)} g(x-2 p \mu+n, y-2 p v) .
$$

The right action of $D_{\mu, \nu}^{c}$ is given by

$$
(f \cdot F)(x, y)=\sum_{q} f(x-2 q \mu, y-2 q v) F(-q, x-2 q \mu, y-2 q v) .
$$

Moreover $\operatorname{Tr}\left(\operatorname{Id}_{N_{\mu, v}^{c}}\right)=2 \mu$. 
We will write $N$ instead of $N_{\mu, v}^{c}$ whenever possible.

Remark 4.4. This module $N$ is in fact "dual" to the module $X$ given in [2], p. 2.

We will see later (Definitions 4.15, 4.11 and 4.18) that we can define another module $N^{\dagger}$ based on $N$ and an isomorphism between two QHM, and "smooth versions" $\mathcal{N}$ and $\mathcal{N}^{\dagger}$ of $N$ and $N^{\dagger}$.

We are now ready to state

Theorem 4.5. If $\mu \neq 0 \neq v$, we can define finite projective modules $N$ and $N^{\dagger}$ over $D$, and the values of the pairings are given by

Table 1

\begin{tabular}{|l|c|c|c|}
\cline { 2 - 4 } \multicolumn{1}{c|}{} & $\tau$ & $\varphi_{1,3}$ & $\varphi_{2,3}$ \\
\hline$[\mathcal{D}]$ & 1 & 0 & 0 \\
\hline$[\mathcal{N}]$ & $2 \mu$ & $-i 2 \pi$ & 0 \\
\hline$\left[\mathcal{N}^{\dagger}\right]$ & $2 \nu$ & 0 & $i 2 \pi$ \\
\hline
\end{tabular}

Notice that:

- the first column of this table was computed by B. Abadie in [4];

- the first line is easy to compute directly from Definition 4.1 with $e=1$;

- if $\mu=0$ or $\nu=0$, then the finite projective modules $\mathcal{N}$ and $\mathcal{N}^{\dagger}$ are not both defined. In this case, we have to use the isomorphism between $D_{\mu+1, v+1}^{c}$ and $D_{\mu, v}^{c}$ that was proved by Abadie in [2]. This phenomenon of "disappearing module" may be surprising, but it also happens with "Schwartz modules" in the case of noncommutative tori; see [11], part II, definition above Lemma 54.

4.1. Connections and pairings for $\mathcal{N}$. The definition of a (noncommutative) connection is given in [12], III.3, Definition 5:

Definition 4.6 (Connection). Let $\mathcal{A} \stackrel{\rho}{\rightarrow} \Omega$ be a cycle over $\mathcal{A}$ and $\mathcal{E}$ a finite projective right module over $\mathcal{A}$. A connection $\nabla$ on $\mathcal{E}$ is a linear map $\nabla: \mathcal{E} \rightarrow \mathcal{E} \otimes_{\mathcal{A}} \Omega^{1}$ such that

$$
\nabla(\xi a)=(\nabla \xi) a+\xi \otimes d \rho(a)
$$

for all $\xi \in \mathcal{E}$ and all $a \in \mathcal{A}$.

Notice that in the above definition we do not actually need the trace $\int$ of the cycle. We could equally consider a "graded differential algebra over $\mathcal{A}$ ". 
The following proposition enables us to compute the even pairings using the connections; see [12], III.3, Proposition 8:

Proposition 4.7. Let $\mathcal{E}$ be a finite projective right module over $\mathcal{A}$. Assume that we have a cycle $\left(\Omega, d, \int\right)$ over $\mathcal{A}$.

(1) $\widetilde{\mathcal{E}}=\mathcal{E} \otimes_{\mathcal{A}} \Omega$ is a finite projective $\Omega$-module.

(2) Every connection $\nabla$ can be uniquely extended to a linear map from $\tilde{\mathcal{E}}$ into itself that satisfies

$$
\nabla(\xi \otimes \omega)=(\nabla \xi) \omega+\xi \otimes d \omega .
$$

for all $\xi \in \mathcal{E}, \omega \in \Omega$.

(3) We have

$$
\langle[\mathcal{E}],[\phi]\rangle=\frac{1}{m !} \int \theta^{m},
$$

where

- $n$ is the (even) dimension of the cycle $\Omega$ and $n=2 m$;

- $[\mathcal{E}] \in K_{0}(\mathcal{A})$ is the class of $\mathcal{E}$;

- $\phi$ is the character of $\Omega$;

- $\int$ is the trace of the cycle;

- $\theta$ is the endomorphism of $\widetilde{\mathcal{E}}$ defined by $\theta=\nabla^{2}$.

Definition 4.8 (Covariant action). Let $E$ be a right module over a $\mathrm{C}^{*}$-algebra $A$. A covariant action of a Lie group $G$ on $E$ is a pair $(\alpha, \beta)$, where $\alpha$ and $\beta$ are actions of $G$ on $A$ and $E$, respectively, which satisfy

$$
\beta_{g}(\xi a)=\beta_{g}(\xi) \alpha_{g}(a)
$$

for all $\xi \in E$ and all $a \in A$.

If we have a covariant action on a module, we can define a "smooth version" of the module on the "smooth version" of the algebra; the smooth module is equipped with a connection.

We state this formally:

Proposition 4.9. Let $(\alpha, \beta)$ be a covariant action on a right A-module $E$. The subvector space $\mathcal{E} \subseteq E$ defined by

$$
\mathcal{E}=\left\{\xi \in E \mid g \mapsto \beta_{g}(\xi) \text { is in } C^{\infty}(G \rightarrow E)\right\}
$$

has a natural left action by $\mathcal{A} \subseteq A$ and a connection $\nabla: \mathcal{E} \rightarrow \mathcal{E} \otimes \mathrm{g}^{*}$ over the differential algebra $\Omega_{G}$ given by

$$
\nabla_{X} \xi=\lim _{t \rightarrow 0} \frac{\beta_{e^{t X}}(\xi)-\xi}{t}
$$

for all $X \in \mathrm{g}^{*}$. 
Proof. Let us first check that $\mathcal{E}$ is a module over $\mathcal{A}$ : if $\xi \in \mathcal{E}$, then $\xi a \in \mathcal{E}$ because $g \mapsto \beta_{g}(\xi a)=\beta_{g}(\xi) \alpha_{g}(a)$.

Now we must prove that $\nabla$ is a connection:

$$
\begin{aligned}
\nabla_{X}(\xi a) & =\lim _{t \rightarrow 0} \frac{\beta_{e^{t X}}(\xi a)-\xi a}{t} \\
& =\lim _{t \rightarrow 0} \frac{\beta_{e^{t X}}(\xi) \alpha_{e^{t X}}(a)-\xi a}{t} \\
& =\lim _{t \rightarrow 0} \frac{1}{t}\left(\beta_{e^{t X}}(\xi) \alpha_{e^{t X}}(a)-\xi \alpha_{e^{t X}}(a)+\xi \alpha_{e^{t X}}(a)-\xi a\right) \\
& =\left(\nabla_{X} \xi\right) a+\xi \partial_{X}(a) .
\end{aligned}
$$

Proposition 4.10. If $\mu \neq 0$, there is a covariant action $\beta$ of $H_{1}$ on $N$ given by

$$
\beta_{(r, s, t)}(f)(x, y)=e^{i x \frac{\pi}{\mu}(t+s c(x / 2-r-\mu q))} f(x-r, y-s) .
$$

Proof. On the one hand,

$$
\begin{gathered}
\beta_{(r, s, t)}(f \cdot F)(x, y)=e^{i x \frac{\pi}{\mu}(t+s c(x / 2-r-\mu q))} \sum_{q} f(x-r-2 q \mu, y-s-2 q v) \\
\cdot F(x-r-2 q \mu, y-s-2 q v,-q) .
\end{gathered}
$$

On the other hand,

$$
\begin{gathered}
\left(\beta_{(r, s, t)}(f) \cdot \alpha_{(r, s, t)}(F)\right)(x, y) \\
=\sum_{q} e^{i(x-2 q \mu) \frac{\pi}{\mu}(t+s c((x-2 q \mu) / 2-r-\mu q))} f(x-r-2 q \mu, y-s-2 q v) \\
\quad \cdot e(q(t+c s(x-r-2 q v))) F(x-r-2 q \mu, y-s-2 q v,-q) .
\end{gathered}
$$

We can restrict this to study the phase factor

$$
e^{i(x-2 q \mu) \frac{\pi}{\mu}(t+s c((x-2 q \mu) / 2-r-\mu q))} e^{i 2 \pi q(t+c s(x-r-2 q \nu))}
$$

while ignoring the $e^{i \cdot}$ :

$$
\begin{aligned}
(x- & 2 q \mu) \frac{\pi}{\mu}(t+s c((x-2 q \mu) / 2-r-\mu q))+2 \pi q(t+c s(x-r-2 q v)) \\
= & t\left(\frac{\pi}{\mu}(x-2 q \mu)+2 \pi q\right)+s c\left((x-2 q \mu) \frac{\pi}{\mu}((x-2 q \mu) / 2-r-\mu q)\right. \\
\quad & \quad 2 \pi q(x-r-2 q \mu)) \\
= & t \frac{\pi}{\mu} x-s c r\left(\frac{\pi}{\mu}(x-2 q \mu)+2 \pi q\right) \\
\quad & \quad s c(x-2 q \mu) \frac{\pi}{\mu}((x-2 q \mu) / 2-\mu q+2 q \mu) \\
= & t \frac{\pi}{\mu} x-s c r \frac{\pi}{\mu} x+s c(x-2 q \mu) \frac{\pi}{\mu} x / 2=x \frac{\pi}{\mu}(t+s c(x / 2-r-q \mu)) .
\end{aligned}
$$


Integrating this property into the expression of $\beta_{(r, s, t)}(f) \cdot \alpha_{(r, s, t)}(F)$ gives

$$
\begin{aligned}
\left(\beta_{(r, s, t)}(f) \cdot \alpha_{(r, s, t)}(F)\right)(x, y)= & \sum_{q} e^{i x \frac{\pi}{\mu}(t+c s(x / 2-r-q \mu))} \\
& \cdot f(x-r-2 q \mu, y-s-2 q v) \\
& \cdot F(x-r-2 q \mu, y-s-2 q v,-q) \\
= & \beta_{(r, s, t)}(f \cdot F)(x, y) .
\end{aligned}
$$

Definition 4.11. We denote by $\mathcal{N}$ the $\mathcal{D}$-module of the elements of $N$ that are regular under the action $\beta$ of $H_{1}$. $\mathcal{N}$ is a finite projective module over $\mathcal{D}$.

Using the covariant action on $N$, it is easy to construct connections over $\mathcal{N}$ :

Proposition 4.12. The connections over $\mathcal{N}$ associated to $\varphi_{1,3}$ and $\varphi_{2,3}$, respectively, are

and

$$
(\nabla f)(x, y)=-\frac{\partial f}{\partial x}(x, y) d x+\frac{i \pi}{\mu} x f(x, y) d p
$$

$$
(\nabla f)(x, y)=\left(-\frac{\partial f}{\partial y}+i \pi c x\left(\frac{x}{2 \mu}-q\right) f\right)(x, y) d y+\frac{i \pi}{\mu} x f(x, y) d p .
$$

Proof. This is an obvious application of Propositions 4.9 and 4.10.

Proposition 4.13. If $\mu \neq 0$, the values of the pairings over $\mathcal{N}$ are

$$
\left\langle[\mathcal{N}],\left[\varphi_{1,3}\right]\right\rangle=-i 2 \pi, \quad\left\langle[\mathcal{N}],\left[\varphi_{2,3}\right]\right\rangle=0 .
$$

Proof. First consider the case of $\varphi_{1,3}$. Computing $\nabla^{2} f$ yields

$$
\begin{aligned}
\nabla(\nabla f) & =\nabla\left(-\frac{\partial f}{\partial x} d x+\frac{i \pi}{\mu} x f d p\right) \\
& =\left(\frac{\partial^{2} f}{\partial x^{2}} d x-\frac{i \pi}{\mu} x \frac{\partial f}{\partial x} d p\right) d x+\frac{i \pi}{\mu}\left(-\left(f+x \frac{\partial f}{\partial x}\right) d x+\frac{i \pi}{\mu} x^{2} f d p\right) d p \\
& =-i \frac{\pi}{\mu} f \otimes d x \wedge d p .
\end{aligned}
$$

Thus, $\nabla^{2}=-i \frac{\pi}{\mu} \operatorname{Id}_{\mathcal{N}} \otimes d x \wedge d p$. The trace of $\operatorname{Id}_{N}$ was computed by Abadie (Theorem 4.3). The pairing is given by Table 1: $\tau(p)=2 \mu$. Hence

$$
\left\langle[\mathcal{N}], \varphi_{1,3}\right\rangle=-i 2 \pi \text {. }
$$

In the case of $\varphi_{2,3}$, the two terms in the connection commute, so $\nabla^{2}=0$ and

$$
\left\langle[\mathcal{N}], \varphi_{2,3}\right\rangle=0 .
$$


4.2. Connections and pairings for $\mathcal{N}^{\dagger}$. We define a second module $N^{\dagger}$ over $D$ through an isomorphism between two $D$ 's:

Proposition 4.14. There is an isomorphism $\Phi: D_{\mu, \nu}^{c} \rightarrow D_{\nu, \mu}^{c}$, induced by the Hilbert bimodule representation

$$
\pi(a)(x, y)=a(-y,-x), \quad \mathcal{T}(\xi)(x, y)=e^{i 2 \pi c(y+\mu)(x+v)} \xi(-y,-x) .
$$

Moreover, this isomorphism intertwines the actions of $H_{1}$,

$$
\Phi\left(\alpha_{r, s, t}(F)\right)=\alpha_{-s,-r,-t-c(\mu s+r v-r s)}^{\prime}(\Phi(F)),
$$

where $\alpha$ and $\alpha^{\prime}$ are the actions of $H_{1}$ over $D_{\mu, \nu}^{c}$ and $D_{v, \mu}^{c}$, respectively.

Before starting the proof let us indicate two points.

The intertwining of the actions proves that $\mathcal{D}_{\mu, v}^{c}$ and $\mathcal{D}_{v, \mu}^{c}$ are sent onto one another.

The existence of such isomorphism was proved in [6], Theorem 2.2. However, we here give an explicit isomorphism and specify the intertwining relation.

Proof. Following Definition 2.5 the algebra $D_{v, \mu, 0}^{c}$ of continuous functions $\mathbb{Z} \times \mathbb{R} \times$ $\mathbb{R} \rightarrow \mathbb{C}$ satisfying

$$
F(p, x+1, y)=e(-c p(y-p \mu)) F(p, x, y), \quad F(p, x, y+1)=F(p, x, y)
$$

is dense in $D_{v, \mu}^{c}$. Definition 2.5 implies that it suffices to check that there is a representation of the Hilbert bimodule $M_{\mu, \nu}^{c}$ in $D_{v, \mu}^{c}$ to obtain the morphism of algebra $\Phi$

Let us check that $\mathcal{T}(\xi)$ has the degree -1 in $D_{v, \mu, 0}^{c}$ :

$$
\begin{aligned}
\mathcal{T}(\xi)(x+1, y) & =e^{i 2 \pi c(y+\mu)(x+1+v)} \xi(-y,-x-1) \\
& =e^{i 2 \pi c(y+\mu)} \mathcal{T}(\xi)(x, y)
\end{aligned}
$$

and

$$
\begin{aligned}
\mathcal{T}(\xi)(x, y+1) & =e^{i 2 \pi c(y+1+\mu)(x+v)} \xi(-y-1,-x) \\
& =e^{i 2 \pi c(x+v)} e^{i 2 \pi c(y+\mu)(x+v)} e^{i 2 \pi c(-x-v)} \xi(-y,-x) \\
& =\mathcal{T}(\xi)(x, y) .
\end{aligned}
$$

To show that $\pi$ and $\mathcal{T}$ induce a bimodule representation, it suffices to prove points (i) and (iv) of Definition 2.2. The others are consequences of these two (see [24], Definition 2.1).

Regarding point (i):

$$
\begin{aligned}
& \mathcal{T}(\xi)^{*} \mathcal{T}(\zeta)(0, x, y) \\
& =e^{-i 2 \pi c(y-\mu)(x-v)} \overline{\xi(-y+2 \mu,-x+2 v)} e^{i 2 \pi c(y-\mu)(x-v)} \zeta(-y+2 \mu,-x+2 v) \\
& =\overline{\xi(-y+2 \mu,-x+2 v)} \zeta(-y+2 \mu,-x+2 v) \\
& =\pi\left(\langle\xi, \zeta\rangle_{A}\right)(x, y) .
\end{aligned}
$$


As for point (iv):

$$
\begin{aligned}
\mathcal{T}(\zeta) \mathcal{T}(\xi)^{*}(0, x, y) & =e^{i 2 \pi c(y+\mu)(x+v)} \zeta(-y,-x) e^{-i 2 \pi c(y+\mu)(x+v) \overline{\xi(-y,-x)}} \\
& =\zeta(-y,-x) \overline{\xi(-y,-x)} \\
& =\pi\left({ }_{A}\langle\zeta, \xi\rangle\right)(x, y) .
\end{aligned}
$$

Hence there is a homomorphism from $D_{\mu, \nu}^{c}$ into $D_{\nu, \mu}^{c}$.

The mappings $\pi(A)$ and $\mathcal{T}(E)$ generate the algebra, hence it is sufficient to check the intertwining (4.1) on these two sets. Denoting by $\alpha^{\prime}$ the action of $H_{1}$ on $D_{\nu, \mu}^{c}$, we get

$$
\begin{aligned}
\pi\left(\alpha_{r, s, t}(a)\right)(x, y) & =\pi(a(x-r, y-s)) \\
& =a(-y-r,-x-s) \\
& =\alpha_{-s,-r,-t-c(\mu s+r v-r s)}^{\prime}(\pi(a))(x, y)
\end{aligned}
$$

as well as

$$
\begin{aligned}
\mathcal{T} & \left(\alpha_{r, s, t} \xi\right)(x, y) \\
= & \mathcal{T}(e(-(t+c s(x-r))) \xi(x-r, y-s)) \\
= & e^{i 2 \pi c(y+\mu)(x+v)} e(-(t+c s(-y-r))) \xi(-y-r,-x-s) \\
= & e(-t+c(y+\mu+r)(x+v+s)-c r(x+v+s) \\
& \quad-c(y+\mu) s+c s(y+r)) \xi(-y-r,-x-s) \\
= & e(-t+c(y+\mu+r)(x+v+s)-c r(x+v)-c \mu s) \xi(-y-r,-x-s) \\
= & e(-t-c(\mu s+r v-r s)-c s(x+r))) e^{i 2 \pi c(y+\mu+r)(x+v+s)} \xi(-y-r,-x-s) \\
= & \left(\alpha_{-s,-r,-t-c(\mu s+r v-r s)}^{\prime} \mathcal{T}(\xi)\right)(x, y) .
\end{aligned}
$$

Definition 4.15 (Module $N_{\mu, v}^{\dagger c}$ ). We define the right module $N_{\mu, \nu}^{\dagger c}$ over $D_{\mu, \nu}^{c}$ as the module induced from $N_{v, \mu}^{c}$ through $\Phi$. This module exists as soon as $v \neq 0$. Explicitly, the action of $D_{\mu, v}^{c}$ is given by $f \cdot F=f \Phi(F)$.

To unclutter notations, we will write $N^{\dagger}$ instead of $N_{\mu, v}^{\dagger c}$ whenever possible.

Remark 4.16. If $P^{\prime}$ is a projector of $M_{n}\left(D_{v, \mu}^{c}\right)$ associated to $N_{v, \mu}^{c}$, then $P=$ $\Phi^{-1}\left(P^{\prime}\right)$ is a projector of $M_{n}\left(D_{\mu, \nu}^{c}\right)$ associated to $N_{\mu, \nu}^{\dagger c}$.

Lemma 4.17. The cyclic cocycles $\varphi_{i, 3}$ induced on $\mathcal{D}_{\mu, v}^{c}$ from the cyclic cocycles $\varphi_{i, 3}^{\prime}$ over $\mathcal{D}_{v, \mu}^{c}$ are given by

$$
\Phi^{*} \varphi_{1,3}^{\prime}=-\varphi_{2,3}, \quad \Phi^{*} \varphi_{2,3}^{\prime}=-\varphi_{1,3} .
$$


Proof. By taking the derivative of (4.1), one obtains

$$
\partial_{1}^{\prime} \circ \Phi=\Phi \circ\left(c \mu \partial_{3}-\partial_{2}\right), \quad \partial_{2}^{\prime} \circ \Phi=\Phi \circ\left(c v \partial_{3}-\partial_{1}\right), \quad \partial_{3}^{\prime} \circ \Phi=-\Phi \circ \partial_{3}
$$

linking the derivations $\partial_{i}^{\prime}$ of $\mathcal{D}_{\nu, \mu}^{c}$ and $\partial_{i}$ of $\mathcal{D}_{\mu, \nu}^{c}$. The traces $\tau_{\mu, \nu}^{c}$ and $\tau_{v, \mu}^{c}$ are invariant in the sense that $\tau_{v, \mu}^{c}(\Phi(F))=\tau_{\mu, \nu}^{c}(F)$. Consequently, the induced cocycles are

$$
\begin{aligned}
\Phi^{*} \varphi_{i, 3}^{\prime}\left(a_{0}, a_{1}, a_{2}\right) & =\tau\left(\Phi\left(a_{0}\right)\left[\partial_{i}^{\prime}\left(\Phi\left(a_{1}\right)\right) \partial_{3}^{\prime}\left(\Phi\left(a_{2}\right)\right)-\partial_{3}^{\prime}\left(\Phi\left(a_{1}\right)\right) \partial_{i}^{\prime}\left(\Phi\left(a_{2}\right)\right]\right)\right. \\
& =\tau\left(\Phi\left[a_{0}\left(k_{i} \partial_{3}-\partial_{j}\right)\left(a_{1}\right) \partial_{3}\left(a_{2}\right)-\partial_{3}\left(a_{1}\right)\left(k_{i} \partial_{3}-\partial_{j}\right)\left(a_{2}\right)\right]\right) \\
& =-\varphi_{j, 3}\left(a_{0}, a_{1}, a_{2}\right),
\end{aligned}
$$

where $(i, j)$ is a permutation of $(1,2)$ and $k_{1}=c \mu, k_{2}=c v$.

It is easy but fastidious to check that $(r, s, t) \mapsto \alpha_{-s,-r,-t-c(\mu s+r \nu-r s)}^{\prime}$ is indeed a representation of $H_{1}$ on $D_{v, \mu}^{c}$. It then follows from Propositions 4.10, 4.14 and Definition 4.15 that $N^{\dagger}$ can be equipped with a covariant action $\left(\alpha, \beta^{\dagger}\right)$ defined by

$$
\beta_{(r, s, t)}^{\dagger}(f)=\beta_{-s,-r,-t-c(\mu s+r v-r s)}^{\prime}(f),
$$

where $\beta^{\prime}$ is the action $H_{1} \curvearrowright N_{v, \mu}^{c}$ given in Proposition 4.10. This justifies

Definition 4.18. We denote by $\mathcal{N}^{\dagger}$ the $\mathcal{D}$-module of the elements of $N^{\dagger}$ that are regular under the action $\beta^{\dagger}$ of $H_{1} . \mathcal{N}^{\dagger}$ is a finite projective module over $\mathcal{D}$.

Proposition 4.19. If $v \neq 0 \neq \mu$, the pairings of $\mathcal{N}^{\dagger}$ are given by

$$
\left\langle\left[\mathcal{N}^{\dagger}\right], \varphi_{1,3}\right\rangle=0, \quad\left\langle\left[\mathcal{N}^{\dagger}\right], \varphi_{2,3}\right\rangle=i 2 \pi .
$$

Proof. Considering Remark 4.16, the definition of $\Phi^{*} \varphi_{i, 3}$ and Lemma 4.17, we clearly have

$$
\left\langle\left[\mathcal{N}_{\mu, \nu}^{\dagger c}\right], \varphi_{j, 3}\right\rangle=-\left\langle\left[\mathcal{N}_{\mu, \nu}^{\dagger c}\right], \Phi^{*} \varphi_{i, 3}^{\prime}\right\rangle=-\left\langle\left[\mathcal{N}_{v, \mu}^{c}\right], \varphi_{i, 3}\right\rangle,
$$

where $(i, j)$ is a permutation of $(1,2)$. Since Proposition 4.13 gives us the values of the pairings $\left\langle\left[\mathcal{N}_{v, \mu}^{c}\right], \varphi_{1,3}^{\prime}\right\rangle$ and $\left\langle\left[\mathcal{N}_{v, \mu}^{c}\right], \varphi_{2,3}^{\prime}\right\rangle$, we get

$$
\left\langle\left[\mathcal{N}^{\dagger}\right], \varphi_{1,3}\right\rangle=0, \quad\left\langle\left[\mathcal{N}^{\dagger}\right], \varphi_{2,3}\right\rangle=i 2 \pi .
$$

\section{Unitaries and first odd pairings}

The inclusion $A \hookrightarrow D_{\mu, v}^{c}$ induces two elements of $K_{1}\left(D_{\mu, \nu}^{c}\right)$. Using $e(x)=e^{i 2 \pi x}$, we can write

$$
U_{1}(p, x, y)=\delta_{0, p} e(x), \quad U_{2}(p, x, y)=\delta_{0, p} e(y) .
$$

We want to construct a third unitary. As a first step, we extend (2.2) to describe how elements of $M_{\mu, \nu}^{c}$ and their adjoints commute. 
Lemma 5.1. $\sigma\left(\xi^{*} \zeta\right)=\zeta \xi^{*}$ for all $\xi, \zeta \in M_{\mu, \nu}^{c} \subseteq D_{\mu, v}^{c}$.

Proof. We know from (2.2) that $a \xi=\xi \sigma(a)$ for all $a \in A$. Using the elements $\xi_{1}$, $\xi_{2}$ of Proposition 2.6, we can write

$$
\sigma\left(\xi^{*} \zeta\right)\left(\xi_{1} \xi_{1}^{*}+\xi_{2} \xi_{2}^{*}\right)=\xi_{1} \xi^{*} \zeta \xi_{1}^{*}+\xi_{2} \xi^{*} \zeta \xi_{2}^{*}=\zeta \xi_{1}^{*} \xi_{1} \xi^{*}+\zeta \xi_{2}^{*} \xi_{2} \xi^{*}=\zeta \xi^{*},
$$

using the commutativity of $A$.

Remark 5.2. The automorphism $\sigma$ of $A$ can be extended to an automorphism of $D$, also denoted by $\sigma$. We need only set $\sigma=\alpha_{(2 \mu, 2 v, 0)}$.

Proposition 5.3. Let

$$
M_{+}=\left(\begin{array}{cc}
\xi_{1} & 0 \\
-\xi_{2} & 0
\end{array}\right), \quad M_{-}=\left(\begin{array}{cc}
0 & \sigma\left(\xi_{2}\right)^{*} \\
0 & \sigma\left(\xi_{1}\right)^{*}
\end{array}\right)
$$

We get the relations

$$
\begin{array}{ll}
M_{+} M_{+}^{*}=P_{+}=\left(\begin{array}{cc}
\xi_{1} \xi_{1}^{*} & -\xi_{1} \xi_{2}^{*} \\
-\xi_{2} \xi_{1}^{*} & \xi_{2} \xi_{2}^{*}
\end{array}\right), & M_{+}^{*} M_{+}=Q_{+}=\left(\begin{array}{ll}
1 & 0 \\
0 & 0
\end{array}\right), \\
M_{-} M_{-}^{*}=P_{-}=\left(\begin{array}{cc}
\sigma\left(\xi_{2}^{*} \xi_{2}\right) & \sigma\left(\xi_{2}^{*} \xi_{1}\right) \\
\sigma\left(\xi_{1}^{*} \xi_{2}\right) & \sigma\left(\xi_{1}^{*} \xi_{1}\right)
\end{array}\right), & M_{-}^{*} M_{-}=Q_{-}=\left(\begin{array}{ll}
0 & 0 \\
0 & 1
\end{array}\right)
\end{array}
$$

and

$$
\begin{gathered}
P_{ \pm}=P_{ \pm}^{2}=P_{ \pm}^{*}, \quad P_{+}+P_{-}=I_{2}, \quad Q_{ \pm}=Q_{ \pm}^{2}=Q_{ \pm}^{*}, \quad Q_{+}+Q_{-}=I_{2}, \\
P_{ \pm} M_{ \pm}=M_{ \pm}, \quad M_{ \pm} Q_{ \pm}=M_{ \pm}, \quad M_{ \pm} M_{\mp}^{*}=0, \quad M_{ \pm}^{*} M_{\mp}=0 .
\end{gathered}
$$

Thus $U_{3}=M_{+}+M_{-}$is a unitary.

We will compute the index of $U_{3}$ in Proposition 7.1.

Proof. The first four relations are proved by direct calculation. Those involving $Q_{ \pm}$ are obvious because of (2.3). The $P_{ \pm}$are clearly self-adjoint. A direct computation using $\xi_{1} \xi_{1}^{*}+\xi_{2} \xi_{2}^{*}=\sum_{i=1}^{2}\left\langle\xi_{i}, \xi_{i}\right\rangle=1$ shows that $P_{-}^{2}=P_{-}$.

Let us now show that $P_{+}+P_{-}=I_{2}$, which proves the relations between $P_{ \pm}$:

$$
\begin{aligned}
\left(\begin{array}{cc}
\xi_{1} \xi_{1}^{*} & -\xi_{1} \xi_{2}^{*} \\
-\xi_{2} \xi_{1}^{*} & \xi_{2} \xi_{2}^{*}
\end{array}\right)+\left(\begin{array}{cc}
\sigma\left(\xi_{2}^{*} \xi_{2}\right) & \sigma\left(\xi_{2}^{*} \xi_{1}\right) \\
\sigma\left(\xi_{1}^{*} \xi_{2}\right) & \sigma\left(\xi_{1}^{*} \xi_{1}\right)
\end{array}\right) & =\left(\begin{array}{cc}
\xi_{1} \xi_{1}^{*}+\xi_{2} \xi_{2}^{*} & -\xi_{1} \xi_{2}^{*}+\xi_{1} \xi_{2}^{*} \\
-\xi_{2} \xi_{1}^{*}+\xi_{2} \xi_{1}^{*} & \xi_{2} \xi_{2}^{*}+\xi_{1} \xi_{1}^{*}
\end{array}\right) \\
& =I_{2},
\end{aligned}
$$

by a systematic use of Lemma 5.1. Finally,

$$
\left(M_{ \pm} Q_{ \pm}-M_{ \pm}\right)^{*}\left(M_{ \pm} Q_{ \pm}-M_{ \pm}\right)=\left(Q_{ \pm} M_{ \pm}^{*}-M_{ \pm}^{*}\right)\left(M_{ \pm} Q_{ \pm}-M_{ \pm}\right)=0
$$

ensures that $M_{ \pm} Q_{ \pm}=M_{ \pm}$. Now $P_{ \pm} M_{ \pm}=M_{ \pm} M_{ \pm}^{*} M_{ \pm}=M_{ \pm} Q_{ \pm}=M_{ \pm}$, and

$$
M_{ \pm} M_{\mp}^{*}=M_{ \pm} Q_{ \pm} Q_{\mp} M_{\mp}^{*}=0, \quad M_{ \pm}^{*} M_{\mp}=M_{ \pm}^{*} P_{ \pm} P_{\mp} M_{\mp}=0 .
$$


The definition of odd pairings can be found in [12], III.3, Proposition 3, p. 224:

Definition 5.4 (Chern-Connes pairings). The following formula defines a bilinear pairing between $K_{1}(\mathcal{A})$ and $\mathrm{HC}^{n}(\mathcal{A})$ :

$$
\langle[U],[\phi]\rangle=\frac{2^{-n}}{\sqrt{2 i}} \Gamma\left(\frac{n}{2}+1\right)^{-1}(\phi \# \operatorname{Tr})\left(U^{*}-1, U-1, U^{*}-1, \ldots, U-1\right) .
$$

Here $n=2 m+1,[U] \in K_{1}(\mathcal{A})$ and $\phi \in Z C^{2 m+1}(\mathcal{A})$. These pairings satisfy $\langle[U],[S \phi]\rangle=\langle[U],[\phi]\rangle$.

Theorem 5.5. Using the previous unitaries $U_{i}$, the values of the pairings are given by

Table 2

\begin{tabular}{|c|c|c|c|}
\cline { 2 - 4 } \multicolumn{1}{c|}{} & $\varphi_{1}$ & $\varphi_{2}$ & $\varphi_{1,2,3}$ \\
\hline$\left[U_{1}\right]$ & $-\sqrt{i 2 \pi}$ & 0 & 0 \\
\hline$\left[U_{2}\right]$ & 0 & $-\sqrt{i 2 \pi}$ & 0 \\
\hline$\left[U_{3}\right]$ & $-\sqrt{i 2 \pi} 2 c \nu$ & $\sqrt{i 2 \pi} 2 c \mu$ & $-(i 2 \pi)^{3 / 2} c / 3$ \\
\hline
\end{tabular}

The proof of these results will occupy the next two sections.

Proposition 5.6. The pairings with $U_{1}$ and $U_{2}$ always vanish, except

$$
\left\langle\left[U_{1}\right],\left[\varphi_{1}\right]\right\rangle=-\sqrt{i 2 \pi}, \quad\left\langle\left[U_{2}\right],\left[\varphi_{2}\right]\right\rangle=-\sqrt{i 2 \pi} .
$$

Proof. For $i=1,2$ and $j=1,2,3$, let us evaluate

$$
\left\langle\left[U_{i}\right],\left[\varphi_{j}\right]\right\rangle=\frac{1}{\sqrt{2 i \pi}} \tau\left(\left(U_{i}^{*}-1\right) \partial_{j}\left(U_{i}-1\right)\right)=\frac{1}{\sqrt{2 i \pi}} \tau\left(U_{i}^{*} \partial_{j}\left(U_{i}\right)\right),
$$

using $\tau\left(\partial_{j}(X)\right)=0$ and $\partial_{j}(1)=0$. The explicit expressions of $\partial_{j}$ in degree 0 and $U_{i}$ yield

$$
\left\langle\left[U_{i}\right],\left[\varphi_{j}\right]\right\rangle=-\delta_{i, j} \sqrt{2 i \pi}
$$

The pairings with $\varphi_{1,2,3}$ are given by

$$
\left\langle\left[U_{i}\right], \varphi_{1,2,3}\right\rangle=\sum_{\sigma \in \Sigma_{3}} \varepsilon(\sigma) \tau\left(\left(U_{i}^{*}-1\right) \partial_{\sigma(1)}\left(U_{i}-1\right) \partial_{\sigma(2)}\left(U_{i}^{*}-1\right) \partial_{\sigma(3)}\left(U_{i}-1\right)\right) .
$$

Each term of the above sum contains a vanishing derivation, so $\left\langle\left[U_{i}\right], \varphi_{1,2,3}\right\rangle=0$. 
To evaluate the other odd pairings, we need to estimate the commutation relations between $\alpha$ and the derivations $\partial_{i}$.

Notation 5.7. We use the notation $\partial_{(u, v, w)}=u \partial_{1}+v \partial_{2}+w \partial_{3}$.

Proposition 5.8. The equality

$$
\alpha_{r, s, t}\left(\partial_{(u, v, w)}(F)\right)=\partial_{\left(u^{\prime}, v^{\prime}, w^{\prime}\right)}\left(\alpha_{r, s, t}(F)\right)
$$

is satisfied if and only if

$$
u=u^{\prime}, \quad v=v^{\prime}, \quad w=w^{\prime}+c\left(v^{\prime} r-s u^{\prime}\right) .
$$

Proof. This is a straightforward computation.

Proposition 5.9. The pairings of $U_{3}$ with degree 1 cocycles are

$$
\left\langle\left[U_{3}\right],\left[\varphi_{1}\right]\right\rangle=-\sqrt{i 2 \pi} 2 c v, \quad\left\langle\left[U_{3}\right],\left[\varphi_{2}\right]\right\rangle=\sqrt{i 2 \pi} 2 c \mu, \quad\left\langle\left[U_{3}\right],\left[\varphi_{3}\right]\right\rangle=0 .
$$

Proof. To simplify notations, we write $\partial$ and $\varphi$ instead of $\partial_{i}$ and $\varphi_{i}$. Remember that $U_{3}=M_{+}+M_{-}$:

$$
\sqrt{2 i \pi}\left\langle U_{3}, \varphi\right\rangle=\tau\left(\left(M_{+}^{*}+M_{-}^{*}-1\right) \partial\left(M_{+}+M_{-}\right)\right)=\tau\left(\left(M_{+}^{*}+M_{-}^{*}\right) \partial\left(M_{+}+M_{-}\right)\right)
$$

because $\tau\left(\partial\left(M_{+}+M_{-}\right)\right)=0$. The trace vanishes on nonzero degree elements, hence we keep only the degree 0 terms of the expression:

$$
\sqrt{2 i \pi}\left\langle U_{3}, \varphi\right\rangle=\tau\left(M_{+}^{*} \partial M_{+}+M_{-}^{*} \partial M_{-}\right) .
$$

Explicit computations yield

$$
\begin{aligned}
M_{+}^{*} \partial M_{+} & =\left(\begin{array}{cc}
\xi_{1}^{*} \partial \xi_{1}+\xi_{2}^{*} \partial \xi_{2} & 0 \\
0 & 0
\end{array}\right) \\
M_{-}^{*} \partial M_{-} & =\left(\begin{array}{cc}
0 & 0 \\
0 & \sigma\left(\xi_{2}\right) \partial \sigma\left(\xi_{2}^{*}\right)+\sigma\left(\xi_{1}\right) \partial \sigma\left(\xi_{1}^{*}\right)
\end{array}\right) .
\end{aligned}
$$

The commutation relation $\partial \sigma(F)=\sigma\left(\partial(F)+k \partial_{3}(F)\right)$ holds for all $\partial=\partial_{i}, i \in$ $\{1,2,3\}$, using different constants $k=k_{i}$. Proposition 5.8 ensures that

$$
k_{1}=-2 c v, \quad k_{2}=2 c \mu, \quad k_{3}=0 .
$$

By integrating the commutations relations in the trace of $M_{+}^{*} \partial M_{+}$we obtain

$$
\begin{aligned}
& \sigma\left(\xi_{1}\right) \sigma\left(\partial \xi_{1}^{*}+k \partial_{3}\left(\xi_{1}^{*}\right)\right)+\sigma\left(\xi_{2}\right) \sigma\left(\partial \xi_{2}^{*}+k \partial_{3}\left(\xi_{2}^{*}\right)\right) \\
& \quad=\sigma\left(\xi_{1} \partial\left(\xi_{1}^{*}\right)+\xi_{2} \partial\left(\xi_{2}^{*}\right)+k\left(\xi_{1} \partial_{3}\left(\xi_{1}^{*}\right)+\xi_{2} \partial_{3}\left(\xi_{2}^{*}\right)\right)\right)
\end{aligned}
$$


But $\partial_{3}\left(\xi_{i}^{*}\right)=i 2 \pi \xi_{i}^{*}$ and $\xi_{1} \xi_{1}^{*}+\xi_{2} \xi_{2}^{*}=1$, so

$$
\begin{aligned}
\sqrt{2 i \pi}\left\langle U_{3}, \varphi\right\rangle & =\tau\left(\xi_{1}^{*} \partial \xi_{1}+\xi_{2}^{*} \partial \xi_{2}\right)+\tau\left(\sigma\left(\xi_{1} \partial \xi_{1}^{*}+\xi_{2} \partial \xi_{2}^{*}\right)\right)+i 2 \pi k \\
& =\tau\left(\xi_{1}^{*} \partial \xi_{1}+\xi_{2}^{*} \partial \xi_{2}+\sigma\left(\xi_{1} \partial \xi_{1}^{*}+\xi_{2} \partial \xi_{2}^{*}\right)\right)+i 2 \pi k \\
& =\tau\left(\xi_{1}^{*} \partial \xi_{1}+\xi_{2}^{*} \partial \xi_{2}+\partial\left(\xi_{1}^{*}\right) \xi_{1}+\partial\left(\xi_{2}^{*}\right) \xi_{2}\right)+i 2 \pi k \\
& =\tau\left(\partial\left(\xi_{1}^{*} \xi_{1}+\xi_{2}^{*} \xi_{2}\right)\right)+i 2 \pi k \\
& =\tau(\partial(1))+i 2 \pi k
\end{aligned}
$$

where we applied Lemma 5.1. Taking into account the different values of $k_{i}$ we get

$$
\left\langle U_{3}, \varphi_{1}\right\rangle=-\sqrt{2 i \pi} 2 c v, \quad\left\langle U_{3}, \varphi_{2}\right\rangle=\sqrt{2 i \pi} 2 c \mu, \quad\left\langle U_{3}, \varphi_{3}\right\rangle=0 .
$$

\section{Top degree pairing}

Proposition 6.1. The pairing $\left\langle\left[U_{3}\right], \varphi_{1,2,3}\right\rangle$ is

$$
\left\langle\left[U_{3}\right],\left[\varphi_{1,2,3}\right]\right\rangle=-(i 2 \pi)^{3 / 2} c / 3 \text {. }
$$

The proof of this proposition will fill the remainder of this section. Setting $U=$ $U_{3}=M_{+}+M_{-}$, we want to evaluate

$$
\left\langle\left[U_{3}\right], \varphi_{1,2,3}\right\rangle=\frac{1}{8 \sqrt{2 i}} \frac{4}{3 \sqrt{\pi}} \sum_{\sigma \in \Sigma_{3}} \varepsilon(\sigma) T_{\sigma(1) \sigma(2) \sigma(3)},
$$

where $\varepsilon(\sigma)$ is the signature of the permutation $\sigma$ of $\{1,2,3\}$ and

$$
\begin{aligned}
T_{i j k} & =\tau\left(\left(u^{*}-1\right) \partial_{i}(u-1) \partial_{j}\left(U^{*}-1\right) \partial_{k}(u-1)\right) \\
& =\tau\left(\left(u^{*}-1\right) \partial_{i}(u) \partial_{j}\left(U^{*}\right) \partial_{k}(u)\right) .
\end{aligned}
$$

All the following terms have odd degree and therefore vanishing trace:

$$
\tau\left(\partial_{i}(\mathcal{U}) \partial_{j}\left(\mathcal{U}^{*}\right) \partial_{k}(u)\right)=\tau\left(\partial_{i}\left(M_{+}+M_{-}\right) \partial_{j}\left(M_{+}^{*}+M_{-}^{*}\right) \partial_{k}\left(M_{+}+M_{-}\right)\right)=0 .
$$

Hence

$$
T_{i j k}=\tau\left(u^{*} \partial_{i}(u) \partial_{j}\left(\mathcal{U}^{*}\right) \partial_{k}(u)\right)
$$

Lemma 6.2. The following relations hold:

$$
\begin{aligned}
\partial_{i}\left(M_{ \pm}\right) M_{\mp}^{*} & =-M_{ \pm} \partial_{i}\left(M_{\mp}^{*}\right) \\
\partial_{i}\left(M_{ \pm}^{*}\right) P_{\mp} & =-M_{ \pm}^{*} \partial_{i}\left(P_{\mp}\right) \\
\tau\left(P_{\mp} \partial_{i}\left(M_{ \pm}\right) \partial_{j}\left(M_{ \pm}^{*}\right)\right) & =\tau\left(P_{ \pm} \partial_{j}\left(P_{ \pm}\right) \partial_{i}\left(P_{ \pm}\right)\right) \\
\tau\left(P_{ \pm} \partial_{i}\left(M_{ \pm}\right) \partial_{j}\left(M_{ \pm}^{*}\right)\right) & =\tau\left(\partial_{i}\left(M_{ \pm}\right) \partial_{j}\left(M_{ \pm}^{*}\right)+P_{ \pm} \partial_{j}\left(P_{ \pm}\right) \partial_{i}\left(P_{ \pm}\right)\right) .
\end{aligned}
$$


Beware of permutations between $i$ and $j$ in equations (6.3) and (6.4)!

Proof. The first series can be proved by integration by parts,

$$
\partial_{i}\left(M_{ \pm}\right) M_{\mp}^{*}=\partial_{i}\left(M_{ \pm} M_{\mp}^{*}\right)-M_{ \pm} \partial_{i}\left(M_{\mp}^{*}\right)=-M_{ \pm} \partial_{i}\left(M_{\mp}^{*}\right)
$$

because (Lemma 5.3) $M_{ \pm} M_{\mp}^{*}=0$. We also have $M_{ \pm}^{*} P_{\mp}=M_{ \pm}^{*} P_{ \pm} P_{\mp}=0$, which makes it possible to prove the second equality of (6.2) using the same method. Now

$$
\begin{aligned}
\tau\left(P_{\mp} \partial_{i}\left(M_{ \pm}\right) \partial_{j}\left(M_{ \pm}^{*}\right)\right) & =\tau\left(\partial_{j}\left(M_{ \pm}^{*}\right) P_{\mp} \partial_{i}\left(M_{ \pm}\right)\right) \\
& =-\tau\left(M_{ \pm}^{*} \partial_{j}\left(P_{\mp}\right) \partial_{i}\left(M_{ \pm}\right)\right) \\
& =\tau\left(M_{ \pm}^{*} \partial_{j}\left(P_{ \pm}\right) \partial_{i}\left(M_{ \pm}\right)\right) \\
& =\tau\left(M_{ \pm}^{*} \partial_{j}\left(P_{ \pm}\right)\left(\partial_{i}\left(P_{ \pm}\right) M_{ \pm}+P_{ \pm} \partial_{i}\left(M_{ \pm}\right)\right)\right) \\
& =\tau\left(P_{ \pm} \partial_{j}\left(P_{ \pm}\right) \partial_{i}\left(P_{ \pm}\right)+M_{ \pm}^{*} P_{ \pm} \partial_{j}\left(P_{ \pm}\right) P_{ \pm} \partial_{i}\left(M_{ \pm}\right)\right) \\
& =\tau\left(P_{ \pm} \partial_{j}\left(P_{ \pm}\right) \partial_{i}\left(P_{ \pm}\right)\right),
\end{aligned}
$$

using (6.2), $\partial_{i}\left(P_{\mp}\right)=-\partial_{i}\left(P_{ \pm}\right)$and $P_{ \pm} \partial_{i}\left(P_{ \pm}\right) P_{ \pm}=0$. This last equality is true because $P_{ \pm}$is an idempotent and $\partial_{i}$ a derivation.

Regarding the last equation we have

$$
\begin{aligned}
\tau\left(P_{ \pm}\right. & \left.\partial_{i}\left(M_{ \pm}\right) \partial_{j}\left(M_{ \pm}^{*}\right)\right) \\
& =\tau\left(\left(\partial_{i}\left(P_{ \pm} M_{ \pm}\right)-\partial_{i}\left(P_{ \pm}\right) M_{ \pm}\right) \partial_{j}\left(M_{ \pm}^{*}\right)\right) \\
& =\tau\left(\partial_{i}\left(M_{ \pm}\right) \partial_{j}\left(M_{ \pm}^{*}\right)-\partial_{i}\left(P_{ \pm}\right) M_{ \pm} \partial_{j}\left(M_{ \pm}^{*}\right) P_{ \pm}+\partial_{i}\left(P_{ \pm}\right) M_{ \pm} M_{ \pm}^{*} \partial_{j}\left(P_{ \pm}\right)\right) \\
& =\tau\left(\partial_{i}\left(M_{ \pm}\right) \partial_{j}\left(M_{ \pm}^{*}\right)-P_{ \pm} \partial_{i}\left(P_{ \pm}\right) P_{ \pm} M_{ \pm} \partial_{j}\left(M_{ \pm}^{*}\right)+P_{ \pm} \partial_{j}\left(P_{ \pm}\right) \partial_{i}\left(P_{ \pm}\right)\right) \\
& =\tau\left(\partial_{i}\left(M_{ \pm}\right) \partial_{j}\left(M_{ \pm}^{*}\right)+P_{ \pm} \partial_{j}\left(P_{ \pm}\right) \partial_{i}\left(P_{ \pm}\right)\right)
\end{aligned}
$$

because $M_{ \pm}^{*}=M_{ \pm}^{*} P_{ \pm}$.

We will also need the following lemma.

Lemma 6.3. If $(i, j)=(1,2)$ or $(2,1)$, we have both

$$
\tau\left(\partial_{i}(U) \partial_{j}\left(U^{*}\right)\right)=\tau\left(\partial_{i}\left(M_{+}\right) \partial_{j}\left(M_{+}^{*}\right)+\partial_{i}\left(M_{-}\right) \partial_{j}\left(M_{-}^{*}\right)\right)
$$

and

$$
\tau\left(\partial_{1}(u) \partial_{2}\left(u^{*}\right)-\partial_{2}(U) \partial_{1}\left(u^{*}\right)\right)=0 .
$$

Proof. The first equality is obvious by keeping only the degree 0 terms in

$$
\tau\left(\partial_{i}(u) \partial_{j}\left(U^{*}\right)\right)=\tau\left(\partial_{i}\left(M_{+}+M_{-}\right) \partial_{j}\left(M_{+}^{*}+M_{-}^{*}\right)\right) .
$$

The second one is obtained by integration by parts:

$$
\begin{aligned}
& \tau\left(\partial_{1}(U) \partial_{2}\left(u^{*}\right)-\partial_{2}(u) \partial_{1}\left(u^{*}\right)\right) \\
& \quad=\tau\left(\partial_{1}\left(U \partial_{2}\left(u^{*}\right)\right)-U \partial_{1} \partial_{2}\left(u^{*}\right)-\partial_{2}\left(U \partial_{1}\left(u^{*}\right)\right)+U \partial_{2} \partial_{1}\left(u^{*}\right)\right) \\
& \quad=c \tau\left(U \partial_{3}\left(u^{*}\right)\right)=0,
\end{aligned}
$$

using $\left[\partial_{1}, \partial_{2}\right]=-c \partial_{3}$ and $\left\langle\varphi_{3}, \mathcal{U}\right\rangle=0$. 
6.1.1. Terms $\boldsymbol{T}_{\mathbf{1 3 2}}$ and $\boldsymbol{T}_{\mathbf{2 3 1}}$. Using the identity $\partial_{3}\left(M_{ \pm}^{*}\right)= \pm i 2 \pi M_{ \pm}^{*}$, we can evaluate $T_{231}$ :

$$
\begin{aligned}
T_{231}= & i 2 \pi \tau\left(\left(M_{+}^{*}+M_{-}^{*}\right) \partial_{2}(u)\left(M_{+}^{*}-M_{-}^{*}\right) \partial_{1}(u)\right) \\
= & i 2 \pi \tau\left(M_{+}^{*} \partial_{2}(u) M_{+}^{*} \partial_{1}(u)-M_{+}^{*} \partial_{2}(u) M_{-}^{*} \partial_{1}(u)\right. \\
& \left.+M_{-}^{*} \partial_{2}(u) M_{+}^{*} \partial_{1}(u)-M_{-}^{*} \partial_{2}(u) M_{-}^{*} \partial_{1}(u)\right) .
\end{aligned}
$$

The same reasoning for $T_{132}$ gives

$$
\begin{aligned}
T_{132}= & i 2 \pi \tau\left(\left(M_{+}^{*}+M_{-}^{*}\right) \partial_{1}(\mathcal{U})\left(M_{+}^{*}-M_{-}^{*}\right) \partial_{2}(\mathcal{U})\right) \\
= & i 2 \pi \tau\left(M_{+}^{*} \partial_{1}(u) M_{+}^{*} \partial_{2}(u)-M_{+}^{*} \partial_{1}(u) M_{-}^{*} \partial_{2}(u)\right. \\
& \left.+M_{-}^{*} \partial_{1}(u) M_{+}^{*} \partial_{2}(u)-M_{-}^{*} \partial_{1}(u) M_{-}^{*} \partial_{2}(u)\right) .
\end{aligned}
$$

Taking into account the $\varepsilon(\sigma)$ of (6.1) and the fact that $\tau$ is a trace,

$$
T_{231}-T_{132}=i 4 \pi \tau\left(M_{+}^{*} \partial_{2}(\mathcal{U}) M_{-}^{*} \partial_{1}(\mathcal{U})-M_{-}^{*} \partial_{2}(\mathcal{U}) M_{+}^{*} \partial_{1}(\mathcal{U})\right)
$$

As $U=M_{+}+M_{-}$, we can put both terms in the general form

$$
\tau\left(M_{+}^{*} \partial_{i}\left(M_{+}+M_{-}\right) M_{-}^{*} \partial_{j}\left(M_{+}+M_{-}\right)\right)
$$

Keeping only elements of total degree 0 we obtain

$$
\tau\left(M_{+}^{*} \partial_{i}\left(M_{+}\right) M_{-}^{*} \partial_{j}\left(M_{-}\right)+M_{+}^{*} \partial_{i}\left(M_{-}\right) M_{-}^{*} \partial_{j}\left(M_{+}\right)\right)
$$

The first term integrates by parts:

$$
\left(\partial_{i}\left(M_{+}^{*} M_{+}\right)-\partial_{i}\left(M_{+}^{*}\right) M_{+}\right) M_{-}^{*} \partial_{j}\left(M_{-}\right) .
$$

It vanishes because $\partial_{i}\left(M_{+}^{*} M_{+}\right)=\partial_{i}\left(Q_{+}\right)=0$ and $M_{+} M_{-}^{*}=0$. For the second term,

$$
\begin{aligned}
\tau\left(M_{+}^{*}\left(\partial_{i}(\underbrace{M_{-} M_{-}^{*}}_{=P_{-}})-M_{-} \partial_{i}\left(M_{-}^{*}\right)\right) \partial_{j}\left(M_{+}\right)\right) & =\tau\left(M_{+}^{*} \partial_{i}\left(P_{-}\right) \partial_{j}\left(M_{+}\right)\right) \\
& =-\tau\left(\partial_{i}\left(M_{+}^{*}\right) P_{-} \partial_{j}\left(M_{+}\right)\right) \\
& =-\tau\left(P_{-} \partial_{j}\left(M_{+}\right) \partial_{i}\left(M_{+}^{*}\right)\right) \\
& =-\tau\left(P_{+} \partial_{i}\left(P_{+}\right) \partial_{j}\left(P_{+}\right)\right)
\end{aligned}
$$

applying (6.2), the trace property and then (6.3).

Therefore, the contribution of $T_{132}$ and $T_{231}$ is

$$
T_{231}-T_{132}=-i 4 \pi \tau\left(P_{+}\left(\partial_{2} P_{+} \partial_{1} P_{+}-\partial_{1} P_{+} \partial_{2} P_{+}\right)\right) \text {. }
$$


6.1.2. Terms $\boldsymbol{T}_{\mathbf{1 2 3}}$ and $\boldsymbol{T}_{\mathbf{2 1 3}}$. As $\partial_{3}\left(M_{ \pm}\right)=\mp i 2 \pi M_{ \pm}$, the terms $T_{123}$ and $T_{213}$ can be written

$$
\begin{aligned}
T_{i j 3} & =i 2 \pi \tau\left(\left(M_{+}^{*}+M_{-}^{*}\right) \partial_{i}(U) \partial_{j}\left(U^{*}\right)\left(M_{+}-M_{-}\right)\right) \\
& =i 2 \pi \tau\left(\left(P_{+}-P_{-}\right) \partial_{i}(U) \partial_{j}\left(U^{*}\right)\right) \\
& =i 2 \pi \tau\left(\left(2 P_{+}-I_{2}\right) \partial_{i}(U) \partial_{j}\left(U^{*}\right)\right) .
\end{aligned}
$$

Taking the difference $T_{123}-T_{213}$, then using lemma 6.3, the expression becomes:

$$
T_{123}-T_{213}=-i 4 \pi \tau\left(P_{+}\left(\partial_{1}(\mathcal{U}) \partial_{2}\left(\mathcal{U}^{*}\right)-\partial_{2}(\mathcal{U}) \partial_{1}\left(U^{*}\right)\right)\right) .
$$

Let us study the term

$$
\begin{aligned}
\tau\left(P_{+}\right. & \left.\partial_{i}\left(M_{+}+M_{-}\right) \partial_{j}\left(M_{+}^{*}+M_{-}^{*}\right)\right) \\
& =\tau\left(P_{+}\left(\partial_{i}\left(M_{+}\right) \partial_{j}\left(M_{+}^{*}\right)+\partial_{i}\left(M_{-}\right) \partial_{j}\left(M_{-}^{*}\right)\right)\right) \\
& =\tau\left(\partial_{i}\left(M_{+}\right) \partial_{j}\left(M_{+}\right)+P_{+} \partial_{j}\left(P_{+}\right) \partial_{i}\left(P_{+}\right)+P_{-} \partial_{j}\left(P_{-}\right) \partial_{i}\left(P_{-}\right)\right) \\
& =\tau\left(\partial_{i}\left(M_{+}\right) \partial_{j}\left(M_{+}^{*}\right)+\partial_{j}\left(P_{+}\right) \partial_{i}\left(P_{+}\right)\right),
\end{aligned}
$$

using the equations (6.4) and (6.3), and then $\partial_{i}\left(P_{-}\right) \partial_{j}\left(P_{-}\right)=\partial_{i}\left(P_{+}\right) \partial_{j}\left(P_{+}\right)$. Next,

$$
\tau\left(\partial_{1}\left(P_{+}\right) \partial_{2}\left(P_{+}\right)-\partial_{2}\left(P_{+}\right) \partial_{1}\left(P_{+}\right)\right)=0
$$

because $\tau$ is a trace.

Finally, the contribution of the terms $T_{123}$ and $T_{213}$ is

$$
T_{123}-T_{213}=-i 4 \pi \tau\left(\partial_{1}\left(M_{+}\right) \partial_{2}\left(M_{+}^{*}\right)-\partial_{2}\left(M_{+}\right) \partial_{1}\left(M_{+}^{*}\right)\right) .
$$

\subsubsection{Terms $T_{312}$ and $T_{321}$}

Lemma 6.4. The analogs of Lemma 6.2 hold:

$$
\begin{gathered}
Q_{\mp} \partial_{i} M_{ \pm}^{*}=-\partial_{i}\left(Q_{\mp}\right) M_{ \pm}^{*}=0, \quad Q_{ \pm} \partial_{i} M_{ \pm}^{*}=\partial_{i}\left(M_{ \pm}^{*}\right), \\
Q_{\mp} \partial_{i}\left(M_{ \pm}^{*}\right) \partial_{j}\left(M_{ \pm}\right)=0, \\
Q_{ \pm} \partial_{i}\left(M_{ \pm}^{*}\right) \partial_{j}\left(M_{ \pm}\right)=\partial_{i}\left(M_{ \pm}^{*}\right) \partial_{j}\left(M_{ \pm}\right) .
\end{gathered}
$$

Proof. For the first series of relations,

$$
Q_{\mp} \partial_{i} M_{ \pm}^{*}=\partial_{i}\left(Q_{\mp} M_{ \pm}^{*}\right)-\partial_{i}\left(Q_{\mp}\right) M_{ \pm}^{*}=0
$$

because $Q_{\mp} M_{ \pm}^{*}=Q_{\mp} Q_{ \pm} M_{ \pm}^{*}=0$ and $\partial_{i}\left(Q_{ \pm}\right)=0$. Likewise,

$$
Q_{ \pm} \partial_{i} M_{ \pm}^{*}=\partial_{i}\left(Q_{ \pm} M_{ \pm}^{*}\right)-\partial_{i}\left(Q_{ \pm}\right) M_{ \pm}^{*}=\partial_{i}\left(M_{ \pm}^{*}\right),
$$

due to $Q_{ \pm} M_{ \pm}^{*}=M_{ \pm}^{*}$. For the second series,

$$
Q_{\mp} \partial_{i}\left(M_{ \pm}^{*}\right) \partial_{j}\left(M_{ \pm}\right)=-\partial_{i}\left(Q_{\mp}\right) M_{ \pm}^{*} \partial_{j}\left(M_{ \pm}\right)=0,
$$

using the previous relations. The third relation is obvious starting from the first series of relations. 
The terms $T_{312}$ and $T_{321}$ can be written:

$$
\begin{aligned}
T_{3 i j} & =i 2 \pi \tau\left(\left(M_{+}^{*}+M_{-}^{*}\right)\left(M_{+}-M_{-}\right) \partial_{i}\left(U^{*}\right) \partial_{j}(U)\right) \\
& =i 2 \pi \tau\left(\left(Q_{+}-Q_{-}\right) \partial_{i}\left(U^{*}\right) \partial_{j}(U)\right) \\
& =i 2 \pi \tau\left(\left(2 Q_{+}-I_{2}\right) \partial_{i}\left(U^{*}\right) \partial_{j}(u)\right) .
\end{aligned}
$$

Taking the difference $T_{312}-T_{321}$, then using Lemma 6.3, we get

$$
T_{312}-T_{321}=-i 4 \pi \tau\left(Q_{+}\left(\partial_{1}\left(\mathcal{U}^{*}\right) \partial_{2}(u)-\partial_{2}\left(\mathcal{U}^{*}\right) \partial_{1}(u)\right)\right) .
$$

Let us study the term

$$
\begin{aligned}
\tau\left(Q_{+}\right. & \left.\partial_{i}\left(M_{+}^{*}+M_{-}^{*}\right) \partial_{j}\left(M_{+}+M_{-}\right)\right) \\
& =\tau\left(Q_{+}\left(\partial_{i}\left(M_{+}^{*}\right) \partial_{j}\left(M_{+}\right)+\partial_{i}\left(M_{-}^{*}\right) \partial_{j}\left(M_{-}\right)\right)\right. \\
& =\tau\left(\partial_{i}\left(M_{+}^{*}\right) \partial_{j}\left(M_{+}\right)\right) .
\end{aligned}
$$

The difference can thus be written

$$
T_{312}-T_{321}=-i 4 \pi \tau\left(\partial_{1}\left(M_{+}^{*}\right) \partial_{2}\left(M_{+}\right)-\partial_{2}\left(M_{+}^{*}\right) \partial_{1}\left(M_{+}\right)\right) .
$$

6.1.4. Synthesis and final computation. Forming the synthesis of the studied terms we obtain:

$$
\begin{aligned}
6 \sqrt{2 i \pi}\left\langle\left[U_{3}\right], \varphi_{1,2,3}\right\rangle= & \left(T_{231}-T_{132}+T_{123}-T_{213}+T_{312}-T_{321}\right) \\
= & -i 4 \pi \tau\left(P_{+}\left(\partial_{1} P_{+} \partial_{2} P_{+}-\partial_{2} P_{+} \partial_{1} P_{+}\right)\right) \\
& -i 4 \pi \tau\left(\partial_{1}\left(M_{+}\right) \partial_{2}\left(M_{+}^{*}\right)-\partial_{2}\left(M_{+}\right) \partial_{1}\left(M_{+}^{*}\right)\right) \\
& -i 4 \pi \tau\left(\partial_{1}\left(M_{+}^{*}\right) \partial_{2}\left(M_{+}\right)-\partial_{2}\left(M_{+}^{*}\right) \partial_{1}\left(M_{+}\right)\right) \\
= & -i 4 \pi \tau\left(P_{+}\left(\partial_{1} P_{+} \partial_{2} P_{+}-\partial_{2} P_{+} \partial_{1} P_{+}\right)\right) \\
= & -i 4 \pi\left\langle\left[P_{+}\right],\left[\psi_{1,2}\right]\right\rangle,
\end{aligned}
$$

where $\psi_{1,2}$ is the cyclic cocycle defined on $C^{\infty}\left(T^{2}\right)$ by

$$
\left(a_{0}, a_{1}, a_{2}\right) \longmapsto \int_{T^{2}} a_{0}\left(\partial_{1} a_{1} \partial_{2} a_{2}-\partial_{2} a_{1} \partial_{1} a_{2}\right)(x, y) d x d y .
$$

The right-hand side is a pairing on the algebra $A=C\left(T^{2}\right)$. We can compute it using a connection.

First identify the module of $C\left(T^{2}\right)$ which corresponds to the projector $P_{+}$.

Lemma 6.5. The module $P_{+} A^{2}$ is isomorphic to $\left(M_{0,0}^{-c}\right)_{A}$.

Notice that we only need an identification as a module, not as a bimodule.

Notation 6.6. We denote the elements of $M_{0,0}^{-c}$ by $\zeta^{*}$. More generally, we include $M_{0,0}^{-c}$ into $D_{0,0}^{c}$ in the following computation. 
Proof. To identify the module $M$ associated to $P_{+}$, the simplest way is to interpret $M_{+}^{*}$ and $M_{+}$respectively as maps $P_{+} A^{2} \rightarrow M$ and $M \rightarrow P_{+} A^{2}$. Formally, we introduce the maps $\Phi: P_{+} A^{2} \rightarrow M_{0,0}^{-c}$ and $\Psi: M_{0,0}^{-c} \rightarrow P_{+} A^{2}$ :

$$
\Phi\left(\begin{array}{l}
a_{1} \\
a_{2}
\end{array}\right)=\left(\xi_{1}^{*}-\xi_{2}^{*}\right)\left(\begin{array}{l}
a_{1} \\
a_{2}
\end{array}\right), \quad \Psi\left(\zeta^{*}\right)=\left(\begin{array}{c}
\xi_{1} \\
-\xi_{2}
\end{array}\right) \zeta^{*} .
$$

Using the properties of $M_{+}$and $M_{+}^{*}$, we see that the maps $\Phi$ and $\Psi$ are inverse to each another and that they preserve the scalar products.

The following result is well known and can be obtained by using connections:

Proposition 6.7. The pairing of $M^{-c}$ with $\psi_{1,2}$ is given by

$$
\left\langle\left[M_{0,0}^{-c}\right],\left[\psi_{1,2}\right]\right\rangle=i 2 \pi c .
$$

This proposition enables us to complete the computation of $\left\langle\left[U_{3}\right],\left[\varphi_{1,2,3}\right]\right\rangle$ :

$$
\left\langle\left[U_{3}\right],\left[\varphi_{1,2,3}\right]\right\rangle=\frac{-i 4 \pi}{6 \sqrt{i 2 \pi}} i 2 \pi c=-c \frac{(i 2 \pi)^{3 / 2}}{3} .
$$

\section{Consequences of the pairings}

An immediate consequence of (3.2) and Table 2 is that $\left(U_{1}, U_{2}, U_{3}\right)$ forms a basis of $K_{1}(D) \otimes \mathbb{C}$. It is therefore natural to ask if these elements generate $K_{1}(D)$. The answer is no.

To prove the above, first notice that since the QHMs are Pimsner algebras associated to the Hilbert bimodule $M^{c}$, their K-theory fits in the 6-term exact sequence (see [28]):

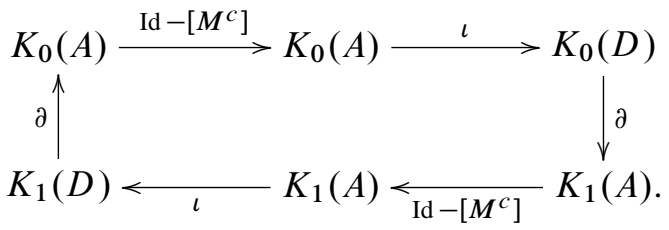

To establish this exact sequence, Pimsner's approach is in fact to start with a short exact sequence

$$
0 \rightarrow J \rightarrow \mathcal{T}_{M} \rightarrow D \rightarrow 0
$$

and to prove that both $J$ and $\mathcal{T}_{M}$ are KK-equivalent to $A$. The algebra $\mathcal{T}_{M}$ was defined in [28]. In our special case, one can prove that $\mathcal{T}_{M}$ can be identified with the *-subalgebra of $\mathcal{T} \otimes D$ generated by $1 \otimes a$ and $S \otimes \xi$ for $a \in A$ and $\xi \in M$. Notice that $\mathcal{T}$ is a nuclear $\mathrm{C}^{*}$-algebra, thus we do not need to specify which $\mathrm{C}^{*}$-norm we 
are using in the tensor product. We identify $\mathcal{T}$ with the unital $\mathrm{C}^{*}$-algebra generated by the projector $P$ and the isometry $S$ with the relations

$$
S S^{*}=1-P, \quad S^{*} S=1 .
$$

To prove that $\left(U_{1}, U_{2}, U_{3}\right)$ does not generate $K_{1}(D)$, we study the images under the index map $\partial: K_{1}(D) \rightarrow K_{0}(A)$.

Proposition 7.1. The index map of the $U_{i}$ is given by

$$
\partial\left(U_{1}\right)=0, \quad \partial\left(U_{2}\right)=0, \quad \partial\left(U_{3}\right)=\left[Q_{-}\right] \ominus\left[P_{+}\right],
$$

with the notation of Proposition 5.3.

Proof. Since $U_{1}$ and $U_{2}$ come from the inclusion $A \hookrightarrow D$, the first two equalities are clear.

Regarding the last equality, we are really going to compute the index map in the 6-term exact sequence associated to (7.2) and then translate the result in terms of K-theory of $A$.

It is readily checked that

$$
\mathbb{U}_{3}=\left(\begin{array}{cc}
S \otimes M_{+}+S^{*} \otimes M_{-} & P \otimes P_{+} \\
P \otimes Q_{-} & S^{*} \otimes M_{+}^{*}+S \otimes M_{-}^{*}
\end{array}\right)
$$

is a unitary lift of $U_{3}$ in $\mathcal{T}_{M}$. It is then easy to complete the index computation:

$$
\begin{aligned}
\mathbb{U}\left(\begin{array}{cc}
1_{2} & 0 \\
0 & 0
\end{array}\right) \mathbb{U}^{*} & \\
= & \left(\begin{array}{cc}
S \otimes M_{+}+S^{*} \otimes M_{-} & 0 \\
P \otimes Q_{-} & 0
\end{array}\right)\left(\begin{array}{cc}
S^{*} \otimes M_{+}^{*}+S \otimes M_{-}^{*} & P \otimes Q_{-} \\
0 & 0
\end{array}\right) \\
\quad= & \left(\begin{array}{cc}
1 \otimes 1-P \otimes P_{+} & 0 \\
0 & P \otimes Q_{-}
\end{array}\right) .
\end{aligned}
$$

Hence, the index of $U_{3}$ in $J$ is $P \otimes Q_{-}-P \otimes P_{+}$, which is the image of $Q_{-} \ominus P_{+}$ in $J$. The KK-equivalence between $A$ and $J$ implies that $\partial\left(U_{3}\right)=Q_{-} \ominus P_{+}$.

In [30], Rieffel has given an explicit description of the finite projective modules over $C\left(T^{2}\right)$. He proves that $K_{0}(A)$ can be identified with the pairs $(d, t)$ where $d$ (dimension) and $t$ (twist) are in $\mathbb{Z}$. We can choose the definition of the twist such that $\left[M^{c}\right] \simeq(1, c)$, and thus $\left[P_{+}\right]=(1,-c)$.

From this identification, together with the Proposition 3.10 of [30], we see that

$$
\mathrm{Id}-[M]:(d, t) \mapsto(d, t)-(d, t+c d)=(0,-c d) .
$$

Thus the kernel of Id $-[M]$ is generated by $(0,1)$. Since $\partial\left(U_{3}\right)=\left[Q_{-}\right] \ominus\left[P_{+}\right] \simeq$ $(0, c),\left(U_{1}, U_{2}, U_{3}\right)$ is not a generator of $K_{1}(D)$.

Another consequence of Proposition 7.1 and the previous computation is 
Corollary 7.2. For any $U \in K_{1}(D)$,

$$
\left\langle[U], \varphi_{1,2,3}\right\rangle=\frac{-\sqrt{i 2 \pi}}{3}\left\langle\partial[U], \psi_{1,2}\right\rangle .
$$

Proof. Since the pairing is bilinear, we only have to check this on a basis of $K_{1}(D) \otimes$ $\mathbb{C}$. The equality is true for $U=U_{1}$ and $U=U_{2}$, since $\partial\left(U_{i}\right)=0$.

For $U_{3}$, notice that $\left\langle\left[Q_{-}\right], \varphi_{1,2,3}\right\rangle=\left\langle[1], \varphi_{1,2,3}\right\rangle=0$. Hence

$$
\left\langle\left[U_{3}\right], \varphi_{1,2,3}\right\rangle=\frac{-i 4 \pi}{6 \sqrt{i 2 \pi}}\left\langle-\left[P_{+}\right], \psi_{1,2}\right\rangle=\frac{-\sqrt{i 2 \pi}}{3}\left\langle\partial\left(U_{3}\right), \psi_{1,2}\right\rangle .
$$

Formula (7.3) enables us to prove a further property of the pairing with $\varphi_{1,2,3}$.

Corollary 7.3. There is a $K$-homology element $K^{\prime} \in K^{1}(D)$ such that, for any $[U] \in K_{1}(D)$,

$$
\left\langle[U], \varphi_{1,2,3}\right\rangle=\frac{(i 2 \pi)^{3 / 2}}{3}\left\langle[U], K^{\prime}\right\rangle .
$$

In particular, this pairing takes only integer values, as one can check by direct examination of Table 2 .

Proof. We know that in $A=C\left(T^{2}\right)$, one can find a $K$-homology element $K$ such that

$$
\left\langle[E], \psi_{1,2}\right\rangle=-i 2 \pi\langle[E], K\rangle
$$

for any $[E] \in K_{0}(A)$. It is known that in the 6-term exact sequence (7.1), one can consider the boundary maps as multiplication by some element $\delta \in \mathrm{KK}^{1}(D, A)$. Multiplying $K \in K^{0}(A)=\mathrm{KK}_{0}(A, \mathbb{C})$ by $\delta$, we get $K^{\prime} \in K^{1}(D)$ such that

$$
i 2 \pi\left\langle[U], K^{\prime}\right\rangle=i 2 \pi\langle\partial[U], K\rangle=-\left\langle\partial[U], \phi_{1,2}\right\rangle=\frac{3}{\sqrt{i 2 \pi}}\left\langle[U], \varphi_{1,2,3}\right\rangle .
$$

\section{Dimension of $\mathrm{HP}^{*}(\mathcal{D})$}

To complete the description of $\operatorname{HP}^{*}(\mathcal{D})$, it only remains to be proven that there are no extra cyclic cocycles. We therefore compute the dimension of $\operatorname{HP}^{0}(\mathcal{D})$ and $\operatorname{HP}^{1}(\mathcal{D})$. We stick to the computation of pairings and periodic theory. We also use results from [20]: in this article it is proved that the smooth QHM $\mathcal{D}$ fits in a short exact sequence of locally convex algebras.

In this section, we denote by $\mathcal{A}$ the algebra $C^{\infty}\left(T^{2}\right)$ with its usual Fréchet algebra structure. We start with the following definitions originally due to Cuntz (see for instance [15], 2.2 and 2.3). 
Definition 8.1 (Smooth Toeplitz algebra). The smooth compact operators $\mathcal{K}$ are the $(\mathbb{N} \times \mathbb{N})$-matrices $\left(a_{i, j}\right)$ with rapidly decreasing complex entries. The topology on $\mathcal{K}$ is given by the norms

$$
p_{n}\left(\left(a_{i, j}\right)\right)=\sum_{i, j}|1+i|^{n}|1+j|^{n}\left|a_{i, j}\right| .
$$

The smooth Toeplitz algebra $\mathcal{T}$ is topologically the direct sum $\mathcal{T}=\mathcal{K} \oplus C^{\infty}\left(S^{1}\right)$, where $C^{\infty}\left(S^{1}\right)$ is equipped with its usual Fréchet structure. Multiplication in $\mathcal{T}$ is described via an action of $\mathcal{T}$ on $\mathcal{S}(\mathbb{N})$ (rapidly decreasing sequences). $\mathcal{K}$ acts in the natural way, and $C^{\infty}\left(S^{1}\right)$ by truncated convolution. The function $\sum a_{k} z^{k}$ acts on $\left(\xi_{i}\right) \in \mathcal{S}(\mathbb{N})$ by

$$
(a \xi)_{i}=\sum_{k+j=i} a_{k} \xi_{j}
$$

The algebra $\mathcal{D}$ fits in the linearly split exact sequence

$$
0 \rightarrow \mathcal{J} \rightarrow \mathcal{T}_{\mathcal{M}} \stackrel{\pi}{\rightarrow} \mathcal{D} \rightarrow 0
$$

where $\mathcal{T}_{\mathcal{M}}$ is the subalgebra generated in the projective tensor product $\mathcal{T} \hat{\otimes} \mathcal{D}$ by $1 \otimes a$, $S \otimes \xi$ and $S^{*} \otimes \xi^{*}$ for $a \in \mathcal{A}$ and $\xi \in \mathcal{M}$ (see [20]). The map $\pi$ is defined on the generators by

$$
\pi(1 \otimes a)=a, \quad \pi(S \otimes \xi)=\xi, \quad \pi\left(S^{*} \otimes \xi^{*}\right)=\xi^{*} .
$$

From this short exact sequence, [16] ensures that there is a 6-term exact sequence in periodic cyclic cohomology:

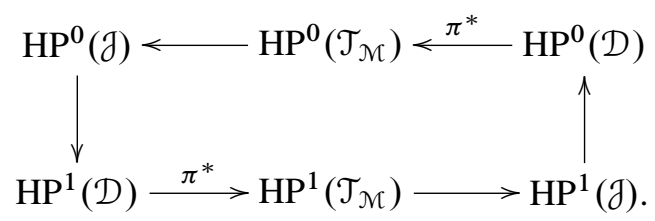

It was also proved in [20] that there are $k k$-equivalences $\mathcal{T}_{\mathcal{M}} \simeq \mathcal{A}$ and $\mathcal{J} \simeq \mathcal{A}$. Consequently

$$
\begin{aligned}
& \operatorname{HP}^{0}(\mathcal{J})=\operatorname{HP}^{0}\left(\mathcal{T}_{\mathcal{M}}\right)=\operatorname{HP}^{0}(\mathcal{A})=\mathbb{C}^{2}, \\
& \operatorname{HP}^{1}(\mathcal{J})=\operatorname{HP}^{1}\left(\mathcal{T}_{\mathcal{M}}\right)=\operatorname{HP}^{1}(\mathcal{A})=\mathbb{C}^{2} .
\end{aligned}
$$

Moreover, the inclusion $\mathcal{A} \hookrightarrow \mathcal{T}_{\mathcal{M}}$ induces an isomorphism at the level of K-theory.

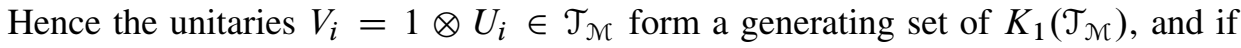
$E$ is a projector in $M_{n}(\mathcal{A})$ with dimension 1 and twisting 1 , then $1 \otimes 1$ and $1 \otimes E$ form a generating set of $K_{0}\left(\mathcal{T}_{\mathcal{M}}\right)$. These generating sets separate the periodic cyclic cocycles.

Let us now evaluate the image of $\pi^{*}$ by computing pairings on $\mathcal{T}_{\mathcal{M}}$. In the odd case,

$$
\left\langle V_{i}, \pi^{*} \varphi_{j}\right\rangle=\left\langle\pi_{*}\left(V_{i}\right), \varphi_{j}\right\rangle=\left\langle U_{i}, \varphi_{j}\right\rangle
$$


which together with (8.2) proves that $\left(\pi^{*} \varphi_{j}\right)_{j=1,2}$ is a basis of $\operatorname{HP}^{1}\left(\mathcal{T}_{\mathcal{M}}\right)$.

In the even case, our analysis of the map Id $-\left[M^{c}\right]$ ensures that in $K_{0}(\mathcal{D}) \iota(E) \ominus$ $\iota(1)$ is a torsion element. Here we used $\iota: \mathcal{A} \hookrightarrow \mathcal{D}$ to distinguish $E \in K_{0}(\mathcal{A})$ from $\iota(E) \in K_{0}(\mathcal{D})$. The definition of $\pi$ guaranties that $\pi(1 \otimes E)=\iota(E)$ and $\pi(1 \otimes 1)=\iota(1)$. The bilinearity of the pairing therefore enforces

$$
\left\langle 1 \otimes E, \pi^{*} \psi\right\rangle=\langle\iota(E), \psi\rangle=\langle\iota(1), \psi\rangle=\left\langle 1 \otimes 1, \pi^{*} \psi\right\rangle .
$$

Since $1 \otimes 1$ and $1 \otimes E$ separate $\operatorname{HP}^{*}(\mathcal{A})$, we see that $\pi^{*}\left(\mathrm{HP}^{0}(\mathcal{D})\right)$ has dimension at most 1 . It is easy to use the trace to check that the image is non zero.

Putting the above results in the exact sequence (8.1), we get:

Proposition 8.2. The periodic cyclic cohomology of $\mathcal{D}$ is given by

$$
\operatorname{HP}^{0}(\mathcal{D})=\mathbb{C}^{3}, \quad \operatorname{HP}^{1}(\mathcal{D})=\mathbb{C}^{3} .
$$

Proposition 3.8, however, provided us with seven cyclic cocycles. Tables 1 and 2 enable us to exhibit linearly independent families of cyclic cocycles. Notice that the pairing $\langle[K],[\varphi]\rangle$ only depends on the class of $\varphi$ in periodic cyclic cohomology and thus the families are independent in $\operatorname{HP}^{*}(\mathcal{D})$. Hence we get:

Theorem 8.3. With the notations of Proposition 3.8, the family $\left(\tau, \varphi_{1,3}, \varphi_{2,3}\right)$ is a basis of $\mathrm{HP}^{0}(\mathcal{D})$, and the family $\left(\varphi_{1}, \varphi_{2}, \varphi_{1,2,3}\right)$ is a basis of $\operatorname{HP}^{1}(\mathcal{D})$.

It follows from [20] that the (tensorized) Chern character is an isomorphism for the QHMs. As we know the K-theory of the QHMs, we get

$$
\mathrm{HP}_{0}(\mathcal{D})=K_{0}(\mathcal{D}) \otimes_{\mathbb{Z}} \mathbb{C}=\mathbb{C}^{3}, \quad \mathrm{HP}_{1}(\mathcal{D})=K_{1}(\mathcal{D}) \otimes_{\mathbb{Z}} \mathbb{C}=\mathbb{C}^{3} .
$$

Since the Chern-Connes pairings factorize through the Chern character from Ktheory to cyclic homology (see [26], Section 8.3), we can describe the periodic cyclic homology. Notice that in the following we require $\mu \neq 0 \neq v$ so that the modules $\mathcal{N}$ and $\mathcal{N}^{\dagger}$ over $\mathcal{D}_{\mu, v}^{c}$ exist (see Definition 4.11 and Definition 4.15).

Theorem 8.4. The family $\left(\mathrm{Ch}\left(U_{1}\right), \mathrm{Ch}\left(U_{2}\right), \mathrm{Ch}\left(U_{3}\right)\right)$ is a basis of $\mathrm{HP}_{1}(\mathcal{D})$.

If $\mu \neq 0 \neq v$, then the family which consists of $\operatorname{Ch}\left(\mathcal{D}_{\mu, v}^{c}\right), \operatorname{Ch}\left(\mathcal{N}_{\mu, v}^{c}\right)$ and $\mathrm{Ch}\left(\mathcal{N}_{\mu, v}^{\dagger c}\right)$ is a basis of $\mathrm{HP}_{0}\left(\mathcal{D}_{\mu, v}^{c}\right)$.

\section{Final remarks}

Note that if we have an element $K$ of $K$-homology, Tables 2 and 1 are precisely what we need to determine the Chern character of $K$ by simply computing its pairings with K-theory. 
The previous discussion of linear independence in $\mathrm{HP}^{*}(\mathcal{D})$ or $\mathrm{HC}^{*}(\mathcal{D})$ is relevant because the cyclic cocycle $\varphi_{3}$ is nonzero in $\operatorname{HC}^{1}(\mathcal{D})$ and $\left(\varphi_{1}, \varphi_{2}, \varphi_{3}\right)$ is linearly independent in $\mathrm{HC}^{1}(\mathcal{D})$, yet $\left[\varphi_{3}\right]=0$ in $\operatorname{HP}^{1}(\mathcal{D})$.

More generally, we have the following proposition which ensures the linear independence of the cyclic cocycles of Proposition 3.8.

Proposition 9.1. The following Hochschild cycles are "dual" to the cocycles of Proposition 3.8 in the sense that

$$
\left\langle c_{k}, \varphi_{i}\right\rangle=\delta_{k, i} k_{i}, \quad\left\langle c_{k, l}, \varphi_{i, j}\right\rangle=\delta_{i, k} \delta_{j, l} k_{i, j}, \quad\left\langle c_{1,2,3}, \varphi_{1,2,3}\right\rangle=c_{1,2,3}
$$

for some nonzero constants $k_{I} \in \mathbb{C}$, where the Hochschild cycles are:

- Degree 1: $c_{1}=U_{1}^{*} \otimes U_{1}, c_{2}=U_{2}^{*} \otimes U_{2}$ and $c_{3}=\xi_{1}^{*} \otimes \xi_{1}+\xi_{2}^{*} \otimes \xi_{2}$.

- Degree 2: “skew-symmetrisation” $c_{j, 3}$ of $\sum_{p} \xi_{p}^{*} U_{j}^{*} \otimes U_{j} \otimes \xi_{p}$ :

$$
c_{j, 3}=\sum_{p=1}^{2} \xi_{p}^{*} U_{j}^{*} \otimes U_{j} \otimes \xi_{p}-U_{j}^{*} \xi_{p}^{*} \otimes \xi_{p} \otimes U_{j}
$$

- Degree 3: setting $U_{p, j}$ equal to $U_{j}$ for $j=1,2$ and equal to $\xi_{p}$ for $j=3$,

$$
c_{1,2,3}=\sum_{p=1}^{2} \sum_{\sigma \in \Sigma_{3}} \varepsilon(\sigma) U_{p, \sigma(3)}^{*} U_{p, \sigma(2)}^{*} U_{p, \sigma(1)}^{*} \otimes U_{p, \sigma(1)} \otimes U_{p, \sigma(2)} \otimes U_{p, \sigma(3)} .
$$

Remark 9.2. The Hochschild cycle $c_{1,2,3}$ is the analog of a fundamental form for $\mathcal{D}$.

Remark 9.3. Cyclic cocycles are Hochschild cocycles and thus the pairings $\left\langle c_{I}, \varphi_{K}\right\rangle$ between Hochschild cycles and cocycles exist. If we consider only Hochschild cocycles, $\varphi_{1,3}, \varphi_{2,3}$ and $\varphi_{1,2}$ are dual in the above sense to $c_{1,3}, c_{2,3}$ and $c_{1,2}$ where $c_{1,2}=U_{1}^{*} U_{2}^{*} \otimes U_{2} \otimes U_{1}-U_{2}^{*} U_{1}^{*} \otimes U_{1} \otimes U_{2}$.

Remark 9.4. The formulas of these cycles are very similar to shuffle products of $c_{i}$. However, shuffle products don't apply directly here, since $\mathcal{D}$ is not commutative.

Proof. It is obvious that $c_{1}$ and $c_{2}$ are Hochschild cycles. It is a straightforward consequence of (2.3) that $c_{3}$ is closed.

To prove that the $c_{i, 3}$ and $c_{1,2,3}$ are Hochschild cycles, we essentially adapt the proof of [21], lemma 12.15. We can apply the same arguments because

$$
e^{-i 4 \pi \mu} U_{1} \xi=\xi U_{1}, \quad e^{-i 4 \pi v} U_{2} \xi=\xi U_{2}
$$

To compensate the fact that $\xi_{p}$ is not unitary, we sum over $p$ and use (2.3). The proof is then a set of lengthy but otherwise straightforward computations. 
We only prove that $c_{1,2,3}$ is a Hochschild cycle and that it pairs non trivially with $\varphi_{1,2,3}$, the other calculations are easier cases of the same thing. We want to evaluate

$$
\begin{aligned}
\left\langle\phi_{1,2,3}, \sum_{i} v_{i}\right\rangle= & \sum_{i} \sum_{\sigma \in \Sigma_{3}} \sum_{\sigma^{\prime} \in \Sigma_{3}} \varepsilon\left(\sigma^{\prime}\right) \varepsilon(\sigma) \\
& \cdot \tau\left(U_{i, \sigma(3)}^{*} U_{i, \sigma(2)}^{*} U_{i, \sigma(1)}^{*} \partial_{\sigma^{\prime}(1)} U_{i, \sigma(1)} \partial_{\sigma^{\prime}(2)} U_{i, \sigma(2)} \partial_{\sigma^{\prime}(3)} U_{i, \sigma(3)}\right) .
\end{aligned}
$$

Given $\sigma$ and $\sigma^{\prime}$, we denote by $T_{\sigma, \sigma^{\prime}}$ the associated term in the above sum. Notice that $\partial_{3}\left(U_{p, j}\right)=\delta_{3, j} i 2 \pi U_{p, j}$. We can find $j_{3}$ such that $\sigma\left(j_{3}\right)=3$. If $\sigma\left(j_{3}\right)=3 \neq$ $\sigma^{\prime}\left(j_{3}\right)$, then $T_{\sigma, \sigma^{\prime}}=0$. As $\partial_{2}\left(U_{p, j}\right)=-\delta_{2, j} i 2 \pi U_{p, j}$, if $\sigma\left(j_{2}\right)=2 \neq \sigma^{\prime}\left(j_{2}\right)$ then $T_{\sigma, \sigma^{\prime}}=0$. Thus $\sigma \neq \sigma^{\prime}$ implies $T_{\sigma, \sigma^{\prime}}=0$. Finally, $\partial_{1}\left(U_{p, j}\right)=-\delta_{1, j} i 2 \pi U_{p, j}$ and

$$
\begin{aligned}
\left\langle\phi_{1,2,3}, \sum_{i} v_{i}\right\rangle & =(i 2 \pi)^{3} \sum_{i} \sum_{\sigma \in \Sigma_{3}} \tau\left(U_{i, \sigma(3)}^{*} U_{i, \sigma(2)}^{*} U_{i, \sigma(1)}^{*} U_{i, \sigma(1)} U_{i, \sigma(2)} U_{i, \sigma(3)}\right) \\
& =(i 2 \pi)^{3} \sum_{i} \sum_{\sigma \in \Sigma_{3}} \tau\left(\xi_{i}^{*} \xi_{i}\right)=(i 2 \pi)^{3} 6 \neq 0,
\end{aligned}
$$

by using the trace property of $\tau$.

About the construction of $U_{3}$ : Proposition 5.3 and Lemma 6.5 essentially show that $U_{3}$ realizes an isomorphism $E^{c} \oplus E^{-c} \simeq 2 E^{0}$, where $E^{t}$ is the line bundle over $T^{2}$ with twisting $t$. Another unitary could be constructed, which would realize the isomorphism $E^{1} \oplus E^{-1} \simeq E^{c-1} \oplus E^{-(c-1)}$. This unitary together with $U_{1}, U_{2}$ would probably be a generator of $K_{1}(D)$. However, the necessary computations are much more involved.

To conclude, on top of its possible implications on noncommutative 3-spheres, our study may foster intuition on Pimsner algebras. In the case of QHMs, the "transfer formula" (7.3) shows how we can "transfer" a pairing from the Pimsner algebra to the basis algebra. This property is very similar to Proposition 12.6 of Nest's article [27]. It would be interesting to investigate whether this is a general phenomenon for Pimsner algebras.

\section{References}

[1] Private communication by J. Renault and slides on A. Kumjian's website. http://wolfweb.unr.edu/homepage/alex/pub/gfest10.pdf

[2] B. Abadie, "Vector bundles" over quantum Heisenberg manifolds. In Algebraic methods in operator theory, Birkhäuser, Boston 1994, 307-315. Zbl 0813.46057 MR 1284956

[3] B. Abadie, Generalized fixed-point algebras of certain actions on crossed products. Pacific J. Math. 171 (1995), 1-21. Zbl 0856.46038 MR 1362977

[4] B. Abadie, The range of traces on quantum Heisenberg manifolds. Trans. Amer. Math. Soc. 352 (2000), 5767-5780. Zbl 0960.46046 MR 1781278 
[5] B. Abadie, S. Eilers, and R. Exel, Morita equivalence for crossed products by Hilbert $C^{*}$-bimodules. Trans. Amer. Math. Soc. 350 (1998), 3043-3054. Zbl 0899.46053 MR 1467459

[6] B. Abadie and R. Exel, Hilbert $C^{*}$-bimodules over commutative $C^{*}$-algebras and an isomorphism condition for quantum Heisenberg manifolds. Rev. Math. Phys. 9 (1997), 411-423. Zbl 0881.46039 MR 1456142

[7] B. Blackadar, Operator algebras - Theory of $C^{*}$-algebras and von Neumann algebras. Encyclopedia Math. Sci. 122, Springer-Verlag, Berlin 2006. Zbl 1092.46003 MR 2188261

[8] L. G. Brown, J. A. Mingo, and N.-T. Shen, Quasi-multipliers and embeddings of Hilbert $C^{*}$-bimodules. Canad. J. Math. 46 (1994), 1150-1174. Zbl 0846.46031 MR 1304338

[9] P. S. Chakraborty, Metrics on the quantum Heisenberg manifold. J. Operator Theory 54 (2005), 93-100. Zbl 1112.46055 MR 2168860

[10] P. S. Chakraborty and K. B. Sinha, Geometry on the quantum Heisenberg manifold. $J$. Funct. Anal. 203 (2003), 425-452. Zbl 1031.46080 MR 2003355

[11] A. Connes, Non-commutative differential geometry. Inst. Hautes Études Sci. Publ. Math. 62 (1985), 41-144. Zbl 0592.46056 MR 0823176

[12] A. Connes, Noncommutative geometry. Academic Press, San Diego, CA, 1994. Zbl 0818.46076 MR 1303779

[13] A. Connes and M. Dubois-Violette, Noncommutative finite-dimensional manifolds. I. Spherical manifolds and related examples. Comm. Math. Phys. 230 (2002), 539--579. Zbl 1026.58005 MR 1937657

[14] A. Connes and M. Dubois-Violette, Noncommutative finite dimensional manifolds. II. Moduli space and structure of noncommutative 3-spheres. Comm. Math. Phys. 281 (2008), 23-127. Zbl 1159.58003 MR 2403605

[15] J. Cuntz, Bivariant $K$-theory and the Weyl algebra. K-Theory 35 (2005), 93-137. Zbl 1111.19003 MR 2240217

[16] J. Cuntz and D. Quillen, Excision in bivariant periodic cyclic cohomology. Invent. Math. 127 (1997), 67-98. Zbl 0889.46054 MR 1423026

[17] S. Doplicher, C. Pinzari, and R. Zuccante, The $C^{*}$-algebra of a Hilbert bimodule. Boll. Unione Mat. Ital. Sez. B Artic. Ric. Mat. (8) 1 (1998), 263-281. Zbl 0916.46053 MR 1638139

[18] R. Exel, A Fredholm operator approach to Morita equivalence. K-Theory 7 (1993), 285-308. Zbl 0792.46051 MR 1244004

[19] N. J. Fowler, P. S. Muhly, and I. Raeburn, Representations of Cuntz-Pimsner algebras. Indiana Univ. Math. J. 52 (2003), 569-605. Zbl 1034.46054 MR 1986889

[20] O. Gabriel and M. Grensing, Six-term exact sequences for smooth generalized crossed products. J. Noncommut. Geom. 7 (2013), 499-524.

[21] J. M. Gracia-Bondía, J. C. Várilly, and H. Figueroa, Elements of noncommutative geometry. Birkhäuser Adv. Texts, Birkhäuser, Boston 2001. Zbl 0958.46039 MR 1789831 
[22] T. Kajiwara, C. Pinzari, and Y. Watatani, Ideal structure and simplicity of the $C^{*}$-algebras generated by Hilbert bimodules. J. Funct. Anal. 159 (1998), 295-322. Zbl 0942.46035 MR 1658088

[23] S. Kang, The Yang-Mills functional and Laplace's equation on quantum Heisenberg manifolds. J. Funct. Anal. 258 (2010), 307-327. Zbl 1186.58007 MR 2557965

[24] T. Katsura, On $C^{*}$-algebras associated with $C^{*}$-correspondences. J. Funct. Anal. 217 (2004), 366-401. Zbl 1067.46054 MR 2102572

[25] E. C. Lance, Hilbert $C^{*}$-modules. London Math. Soc. Lecture Note Ser. 210, Cambridge University Press, Cambridge 1995. Zbl 0822.46080 MR 1325694

[26] J.-L. Loday, Cyclic homology. 2nd ed., Grundlehren Math. Wiss. 301, Springer-Verlag, Berlin 1998. Zbl 0885.18007 MR 1600246

[27] R. Nest, Cyclic cohomology of crossed products with $\mathbb{Z}$. J. Funct. Anal. 80 (1988), 235-283. Zbl 0658.46054 MR 961899

[28] M. V. Pimsner, A class of $C^{*}$-algebras generalizing both Cuntz-Krieger algebras and crossed products by $\mathbb{Z}$. In Free probability theory (Waterloo, ON, 1995), Fields Inst. Commun. 12, Amer. Math. Soc., Providence, RI, 1997, 189-212. Zbl 0871.46028 MR 1426840

[29] C. Pinzari, The ideal structure of Cuntz-Krieger-Pimsner algebras and Cuntz-Krieger algebras over infinite matrices. In Operator algebras and quantum field theory (Rome, 1996), Internat. Press, Cambridge, MA, 1997, 136-150. Zbl 0912.46071 MR 1491114

[30] M. A. Rieffel, The cancellation theorem for projective modules over irrational rotation $C^{*}$-algebras. Proc. London Math. Soc. (3) 47 (1983), 285-302. Zbl 0541.46055 MR 703981

[31] M. A. Rieffel, Deformation quantization of Heisenberg manifolds. Comm. Math. Phys. 122 (1989), 531-562. Zbl 0679.46055 MR 1002830

Received December 13, 2010; revised February 14, 2011

O. Gabriel, Université Denis Diderot Paris 7 - Institut de Mathématiques de Jussieu Équipe algèbres d'opérateurs, 175, rue du Chevaleret, 75013 Paris, France

E-mail: gabriel@math.jussieu.fr 